\title{
GRAVITY SOLITARY WAVES WITH POLYNOMIAL DECAY TO EXPONENTIALLY SMALL RIPPLES AT INFINITY
}

\section{ONDES SOLITAIRES DE GRAVITÉ À DÉCROISSANCE POLYNOMIALE VERS DES OSCILLATIONS EXPONENTIELLEMENT PETITES À L'INFINI}

\author{
E. LOMBARDI ${ }^{\mathrm{a}, *}$, G. IOOSS ${ }^{\mathrm{a}, \mathrm{b}}$ \\ a INLN, UMR CNRS-UNSA 6618 1361, route des Lucioles, 06560 Valbonne, France \\ ${ }^{\mathrm{b}}$ Institut universitaire de France, France
}

Received 3 January 2002, revised 1 May 2002

\begin{abstract}
In this paper, we study the travelling gravity waves of velocity $c$ in a system of two layers of perfect fluids, the bottom one being infinitely deep, the upper one having a finite thickness $h$. We assume that the flow is potential, and the dimensionless parameters are the ratio between densities $\rho=\rho_{2} / \rho_{1}$ and $\lambda=g h / c^{2}$. For $\varepsilon=1-\lambda(1-\rho)$ near $0^{+}$, the existence of periodic travelling waves of arbitrary small amplitude and the existence of generalized solitary waves with ripples at infinity of size larger than $\varepsilon^{5 / 2}$ and polynomial decay rate were established in [7]. In this paper we improve this former result by showing the existence of generalized solitary waves with exponentially small ripples at infinity (of order $\mathcal{O}\left(\mathrm{e}^{-c / \varepsilon}\right)$ ). We conjecture the nonexistence of true solitary waves in this case. The proof is based on a spatial dynamical formulation of the problem combined with a study of the analytic continuation of the solutions in the complex field which enables one to obtain exponentially small upper bounds of the oscillatory integrals giving the size of the oscillations at infinity.

(C) 2003 L'Association Publications de l'Institut Henri Poincaré. Published by Elsevier B.V. All rights reserved
\end{abstract}

Keywords: Nonlinear water waves; Solitary waves with polynomial decay; Bifurcation theory; Infinite dimensional reversible dynamical systems; Normal forms with essential spectrum; Exponentially small effects; Oscillatory integrals

RÉSUMÉ. - Cet article est consacré aux ondes progressives de vitesse $c$ dans un système de deux couches superposées de fluides parfaits en écoulement potentiel, la couche inférieure étant de profondeur infinie, et la supérieure de profondeur finie $h$. Les paramètres sans dimension du problème sont le rapport des densités $\rho=\rho_{2} / \rho_{1}$ et $\lambda=g h / c^{2}$. Pour $\varepsilon=1-\lambda(1-\rho)$ voisin de

\footnotetext{
* Corresponding author.

E-mail addresses: lombardi@inln.cnrs.fr (E. Lombardi), iooss@inln.cnrs.fr (G. Iooss).
} 
$0^{+}$, l'existence d'ondes progressives périodiques de tailles arbitrairement petites et l'existence d'ondes solitaires généralisées à décroissance polynomiale vers des oscillations de taille supérieure à $\varepsilon^{5 / 2}$ ont été obtenues dans [7]. Dans ce présent article nous améliorons ce dernier résultat en montrant l'existence d'ondes solitaires généralisées avec des oscillations résiduelles à l'infini exponentiellement petites ( $\mathrm{d}^{\prime}$ ordre $\left.\mathcal{O}\left(\mathrm{e}^{-c / \varepsilon}\right)\right)$. On conjecture la non existence de vraies ondes solitaires dans ce cas. La démonstration est basée sur une formulation de type "dynamique spatiale" du problème combinée avec une étude du prolongement analytique des solutions qui permet d'obtenir des bornes supérieures exponentiellement petites des intégrales oscillantes donnant la taille des oscillations à l'infini.

(C) 2003 L'Association Publications de l'Institut Henri Poincaré. Published by Elsevier B.V. All rights reserved

\section{Introduction}

Let us consider two layers of perfect fluids (densities $\rho_{1}$ (bottom layer), $\rho_{2}$ (upper layer)), assuming that there is no surface tension, neither at the free surface nor at the interface, and assuming that the flow is potential. The thickness at rest of the upper layer is $h$ while the bottom one has infinite thickness (see Fig. 1). We are interested in travelling waves of horizontal velocity $c$. The dimensionless parameters are $\rho=\rho_{2} / \rho_{1}<1$, and $\lambda=g h / c^{2}$ (inverse of (Froude number) ${ }^{2}$ ).

The existence of a family of periodic travelling waves, for generic values of these parameters is known [6]. This paper is devoted to the problem of existence of solitary waves for $\lambda(1-\rho)$ near $1^{-}$. This problem can be formulated as a spatial reversible dynamical system

$$
\frac{d U}{d x}=F(\rho, \lambda ; U), \quad U(x) \in \mathbb{D},
$$

where $\mathbb{D}$ is an appropriate infinite dimensional Banach space, and where $U=0$ corresponds to a uniform state (velocity $c$ in a moving reference frame). Solitary waves correspond to homoclinic connections to 0 of (1) and generalized solitary waves correspond to homoclinic connections to periodic orbits. A survey of the different results obtained for the water waves problems using a reversible dynamical system approach can be found in [5].

Considering the linearized operator around 0

$$
L_{\varepsilon}=D_{U} F(\rho, \lambda ; 0)
$$

with $\varepsilon=1-\lambda(1-\rho)$, it was shown in [7] that its spectrum contains the entire real line (essential spectrum), with in addition a double eigenvalue in 0 , a pair of simple

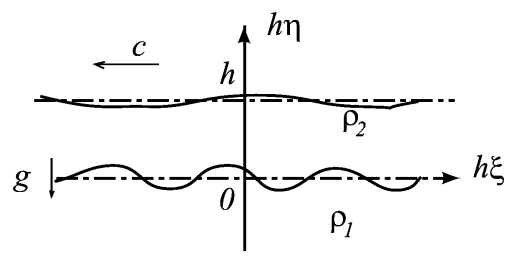

Fig. 1. Two layers, the bottom one being of infinite depth. 


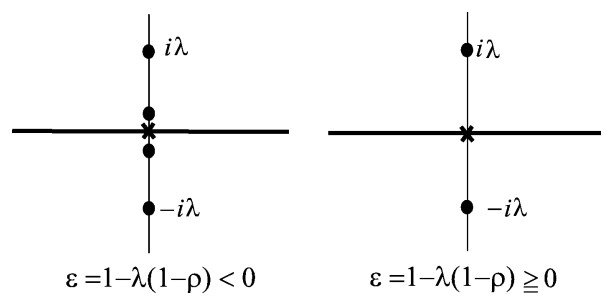

Fig. 2. Spectrum of $L_{\varepsilon}$.
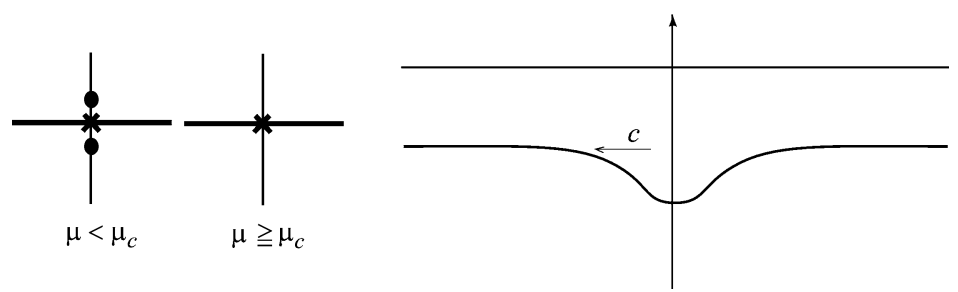

Fig. 3. (Left) $\mathbb{R} 00^{2}$ resonance, and (right) shape of the internal solitary wave in the two-layer system for $\mu>\mu_{c}$ (bottom layer infinitely deep).

imaginary eigenvalues $\pm i \lambda$ at a distance $\mathrm{O}(1)$ from 0 when $\varepsilon$ is near 0 , and for $\varepsilon$ less than 0 , another pair of simple imaginary eigenvalues tending towards 0 as $\varepsilon \rightarrow 0^{-}$. When $\varepsilon \geqslant 0$, this pair completely disappears into the essential spectrum! (see Fig. 2). The rest of the spectrum consists of a discrete set of eigenvalues situated at a distance at least $\mathrm{O}(1)$ from the imaginary axis.

For $\lambda(1-\rho)$ near $1^{-}$, the existence of periodic travelling waves of arbitrary small amplitude induced by the pair of simple purely imaginary eigenvalues $\pm i \lambda$ like in the Lyapunov Devaney Theorem was proved [7] (despite the resonance due to the 0 eigenvalue in the spectrum).

For the solitary waves the situation is more intricate. First we cannot expect the existence of a solitary wave with an exponential decay at infinity because of the lack of spectral gap induced by the existence of the continuous spectrum on the whole real line. We can only expect solitary waves with polynomial decay at infinity. Such solitary waves have been found for two superposed layers, the bottom one being infinitely deep, and the upper one being bounded by a rigid horizontal top, with no interfacial tension (see Fig. 3).

A model equation was derived from the Euler equations thanks to a long-wave approximation of the problem above, by Benjamin [3], Davis and Acrivos [4], and Ono [11]. The now called Benjamin-Ono equation is nonlocal and reads

$$
\mathcal{H}\left(u^{\prime}\right)+u-u^{2}=0
$$

where $\mathcal{H}$ is the Hilbert transform, and $u$ is a scalar function. This equation admits an homoclinic connection to 0 , given explicitly by

$$
u_{h}(\tau)=\frac{2}{1+\tau^{2}}
$$


All the other solutions of Eq. (2) have been described by Amick and Toland [2]. For the full Euler equations, the existence of the solitary waves, with polynomial decay at infinity, has been obtained in this case, independently by Amick [1] and Sun [14]. More precisely, they both proved that, for $\mu>\mu_{c}$ and close to $\mu_{c}$ (we can just play on the velocity $c$ of the wave), the form of the interface for the solitary wave satisfies

$$
Z(x)=\mu u_{h}(\mu x)+\mu^{2} u_{1}(\mu x),
$$

where

$$
\sup _{\tau \in \mathbb{R}}(1+|\tau|)\left|\frac{d^{j} u_{1}}{d \tau^{j}}(\tau)\right| \leqslant K_{j}, \quad j=0,1,2, \ldots
$$

Therefore, the solitary wave solution (3) of the Benjamin-Ono equation (2) gives the first order approximation of a solitary wave solution of the full Euler equations. Neither the approach of Amick, nor the one of Sun was based on a dynamical system approach. However, we observe that the problem may be formulated as a reversible dynamical system, for which the differential at the origin (which corresponds to the rest state) admits the entire real line as essential spectrum, a zero eigenvalue, and a pair of simple imaginary eigenvalues for $\mu<\mu_{c}$ tending towards 0 as $\mu \rightarrow \mu_{c}^{-}$. When $\mu \geqslant \mu_{c}$ this pair completely disappears in the essential spectrum (see Fig. 3).

Observe that for the problem studied in this paper (two layers, the bottom one infinitely deep, no surface tension, no interfacial tension), the behavior of the spectrum of the linearized operator $L_{\varepsilon}$ is the same as the one of the previous example, with in addition an extra pair of simple eigenvalues lying on the imaginary axis, not close to 0 (compare Figs. 2 and 3 ). These additional eigenvalues $\pm i \lambda$ lead to a competition between the oscillatory dynamics they induce, and the Benjamin-Ono type of dynamics induced by the essential spectrum with the 0 eigenvalue. This competition causes the appearance of oscillations at infinity for the solutions. Such a coexistence of an oscillatory dynamics and a hyperbolic dynamics also occurs for one parameter families of reversible vector fields admitting a $0^{2} i \omega$ resonance at the origin, i.e. vector fields admitting the origin as a fixed point and such that the differential at the origin admits the bifurcation of spectrum described on Fig. 4.

For such vector fields it is proved in [10] that there are generically no homoclinic connections to 0 , whereas there are always homoclinic connections to periodic orbits, until they are exponentially small. Such a vector field occurs after a center manifold reduction for one layer of finite depth in presence of gravity and surface tension for a Froude number $\mathrm{F}$ close to 1 , and a Bond number $b$ less than $1 / 3$. In this case, for $\mathrm{F}>1$ and $b<1 / 3$ periodic travelling waves and generalized solitary waves asymptotic at

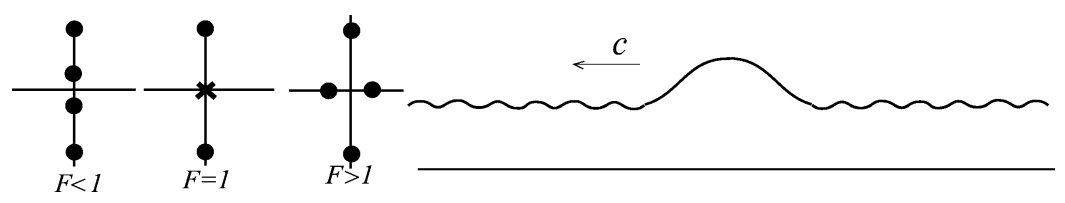

Fig. 4. (Left) $0^{2} i \omega$ resonance, and (right) shape of the generalized solitary waves for $b<1 / 3, \mathrm{~F}>1$. 
infinity to each of these periodic waves, have been obtained provided that the amplitude of the ripples is larger than an exponentially small quantity (as function of $F-1$ ) [13,9] (see Fig. 4). The nonexistence of true solitary waves has also been proved by S.M. Sun [15] for a Froude number $\mathrm{F}$ close to $1^{+}$, and a Bond number $b$ near $1 / 3^{-}$.

We expect a result of the same type here, i.e. nonexistence of true solitary waves and existence of generalized solitary waves with exponentially small ripples at infinity. In [7] a weaker result was obtained, i.e. the existence of homoclinic connections to periodic solutions provided that the size of the limiting periodic orbit is not too small (at least of order $\varepsilon^{5 / 2}$ ). In this paper we show the existence of generalized solitary waves with exponentially small ripples at infinity. The question of the (non)-existence of true solitary waves is still open in this case. We should finally notice that the present problem is numerically studied by Părău and Dias in [12], with lot of information on the shapes of the free surface and interface.

THEOREM 1. - There exists $\delta$ such that for any $\ell \in] 0,1\left[\right.$ there exist $c_{\ell}, \varepsilon_{\ell}>0$, such that for $0<\varepsilon<\varepsilon_{\ell}$, and $c_{\ell} \varepsilon \mathrm{e}^{-\ell \lambda / \varepsilon}<A_{0}<\delta$, (1) has two homoclinic connections $U_{A_{0}, \varepsilon, \varphi_{j}}(j=1,2)$ of the form

$$
U_{A_{0}, \varphi_{j}, \varepsilon}(x)=p_{A_{0}, \varepsilon}\left(x+\varphi_{j} \arctan (\varepsilon x / \rho)\right)-\varepsilon u_{h}(\varepsilon x / \rho) \xi_{0}+\mathcal{O}\left(\frac{\varepsilon^{3 / 2}}{\rho^{2}+\varepsilon|x|}\right),
$$

where $\xi_{0}$ is a fixed vector of $\mathbb{D}, u_{h}$ is the soliton of Benjamin-Ono given by (3) and where $p_{A_{0}, \varepsilon}$ is a periodic function of (1) which reads $p_{A_{0}, \varepsilon}(x)=\hat{p}_{A_{0}, \varepsilon}(s)$ with

$$
\hat{p}_{A_{0}, \varepsilon}(s)=\varepsilon A_{0}\left(\mathrm{e}^{i s} \zeta_{\varepsilon}+\mathrm{e}^{-i s} \bar{\zeta}_{\varepsilon}\right)+\sum_{2 \leqslant p+q \leqslant r+1} \varepsilon^{r+1} A_{0}^{p+q} \mathrm{e}^{i(p-q) s} Y_{p q r}
$$

and $s=(\lambda+\gamma) x$ with $\gamma=\sum_{1 \leqslant n+m+2 p \leqslant r} \gamma_{p r} \varepsilon^{r} A_{0}^{2 p} \in \mathbb{R}$, where coefficients $\zeta_{\varepsilon}, \bar{\zeta}_{\varepsilon}, Y_{p q r}$ lie in $\mathbb{D}$ and $\gamma_{\text {pr }}$ lie in $\mathbb{R}$.

The physical shape of the corresponding generalized solitary waves is given on Fig. 5.

In Section 2 we explain how the problem can be formulated as a spatial dynamical system of the form (1). We also recall the "normal form result" obtained in [7] which states that the full Euler equations (1) are equivalent to a Benjamin-Ono equation, coupled with a nonlinear oscillator equation, with higher order terms.

As already explained the persistence of a pair of simple eigenvalues on the imaginary axis after bifurcation for $\varepsilon>0$ causes the appearance of oscillations at infinity for the

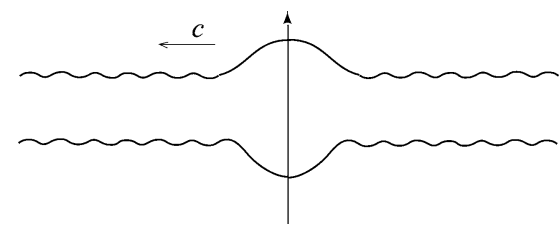

Fig. 5. Shape of generalized solitary waves in the two layer system. 
solutions. The size of the oscillations is given by an oscillatory integrals of the form

$$
I(\varepsilon)=\int_{-\infty}^{+\infty} g(U(s), \varepsilon) \mathrm{e}^{\frac{i \lambda s}{\varepsilon}} d s,
$$

where $g$ is explicitly known whereas $U$ is a solution of (1). In Section 3 we introduce appropriate algebras of holomorphic functions which enables to obtain exponentially small upper bounds of such oscillatory integrals.

Finally, in Section 4 studying the holomorphic continuation of the solutions of (1) we prove Theorem 1.

\section{Formulation as a dynamical system and normal form theory}

\subsection{Dynamical system formulation and scaling}

Travelling waves correspond to stationary solutions in a frame moving with a constant speed $c$. In such a frame the two dimensional travelling waves of Euler equations corresponding to an incompressible potential flow of velocity $\left(u_{j}(\xi, \eta), v_{j}(\xi, \eta)\right)$, in layer $j=1,2$ satisfy

$$
\begin{array}{ll}
\left.\begin{array}{l}
\frac{\partial u_{j}}{\partial \xi}+\frac{\partial v_{j}}{\partial \eta}=0 \\
\frac{\partial u_{j}}{\partial \eta}-\frac{\partial v_{j}}{\partial \xi}=0
\end{array}\right\} & \text { (inside each domain: } j=1,2), \\
u_{2} \widetilde{Z}^{\prime}(\xi)-v_{2}=0 & \text { at } \eta=1+\widetilde{Z}(\xi) \text { (free surface), } \\
u_{2} \widetilde{Z}_{I}^{\prime}(\xi)-v_{2}=u_{1} \widetilde{Z}_{I}^{\prime}(\xi)-v_{1}=0 & \text { at } \eta=\widetilde{Z}_{I}(\xi) \text { (interface), } \\
\frac{1}{2}\left(u_{2}^{2}+v_{2}^{2}\right)+\lambda \widetilde{Z}=\widetilde{c}_{1} & \text { at } \eta=1+\widetilde{Z}(\xi) \text { (free surface), } \\
\frac{1}{2}\left(u_{1}^{2}+v_{1}^{2}\right)-\frac{\rho}{2}\left(u_{2}^{2}+v_{2}^{2}\right)+\lambda(1-\rho) \widetilde{Z}_{I}=\widetilde{c}_{2} & \text { at } \eta=\widetilde{Z}_{I}(\xi) \text { (interface), }
\end{array}
$$

where the parameters are $\rho=\rho_{2} / \rho_{1}<1$, and $\lambda=g h / c^{2}$, and $\widetilde{c}_{1}$ and $\widetilde{c}_{2}$ are arbitrary constants.

In what follows we assume that $\rho$ is fixed and that $\lambda(1-\rho)$ is close to $1^{-}$. So we work with a unique bifurcation parameter $\varepsilon>0$ defined by $\lambda(1-\rho)=1-\varepsilon$.

We define

$$
\lambda_{\varepsilon}=\frac{1-\varepsilon}{1-\rho} \quad \text { and } \quad \lambda_{0}=\frac{1}{1-\rho} .
$$

For formulating our problem into a dynamical system, we first transform the unknown domain into a strip. There are different ways for such a change of coordinates. We choose the one used by Levi-Civita [8]. For that purpose we introduce the complex potential in layer $j$ denoted by $w_{j}(\xi+i \eta)$ and the complex velocity (in dimensionless form) 
$w_{j}^{\prime}(\xi+i \eta)=u_{j}-i v_{j}$. The Euler equations are expressed here by the fact that $w_{j}$ is analytic in $\zeta=\xi+i \eta$. The new unknown are $\alpha_{j}+i \beta_{j}, j=1,2$, which are analytic functions of $w_{j}=x_{j}+i y$, where $x_{j}$ is the velocity potential in the layer $j$, and $y$ the stream function, and where

$$
w_{j}^{\prime}(\xi+i \eta)=\mathrm{e}^{\beta_{j}-i \alpha_{j}},
$$

the free surface is given by $y=1$, and the interface by $y=0$. The region of the flow is $-\infty<y<0$ for fluid 1 , and $0<y<1$ for fluid 2. Observe that the $x$ coordinate given by the Levi-Civita change of coordinate is not the same in each strip. In fact we have $\frac{d x_{2}}{d x_{1}}=\mathrm{e}^{\beta_{20}-\beta_{10}}$ where $\beta_{20}-\beta_{10}$ is the value of $\beta_{2}-\beta_{1}$ taken at the interface $y=0$. So, we choose as the basic $x$ coordinate the one given by the bottom layer $\left(x_{1}\right)$ which then introduces a factor in the Cauchy-Riemann equations of the upper layer. In such a formulation, the unknown is defined by

$$
[U(x)](y)=\left(\beta_{10}(x), \beta_{21}(x), \alpha_{1}(x, y), \beta_{1}(x, y), \alpha_{2}(x, y), \beta_{2}(x, y)\right)^{t}
$$

and the system has the form

$$
\frac{d U}{d x}=F(\varepsilon ; U)
$$

with

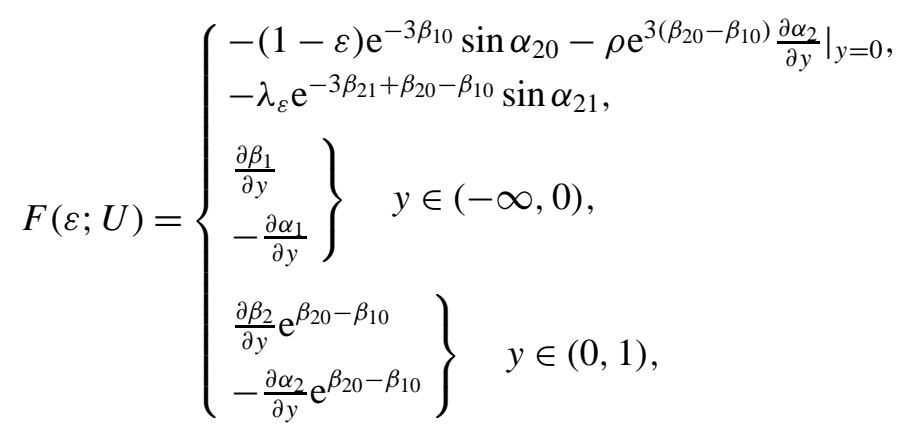

where we denote by $\alpha_{20}, \beta_{10}$ and $\beta_{20}$ the traces of (respectively) $\alpha_{2}, \beta_{1}, \beta_{2}$ at $y=0$, and $\alpha_{21}, \beta_{21}$ the traces of $\alpha_{2}$ and $\beta_{2}$ at $y=1$. Here we choose the basic space

$$
\mathbb{H}=\mathbb{R}^{2} \times C_{1}^{0}\left(\mathbb{R}^{-}\right) \times C_{\lim , 1}^{0}\left(\mathbb{R}^{-}\right) \times\left\{C^{0}(0,1)\right\}^{2}
$$

and the domain of the operator $F$ is:

$$
\begin{aligned}
\mathbb{D}= & \mathbb{R}^{2} \times C_{1}^{1}\left(\mathbb{R}^{-}\right) \times C_{\lim , 1}^{1}\left(\mathbb{R}^{-}\right) \times\left\{C^{1}(0,1)\right\}^{2} \\
& \cap\left\{\alpha_{10}=\alpha_{20}, \beta_{10}=\left.\beta_{1}\right|_{y=0}, \beta_{21}=\left.\beta_{2}\right|_{y=1}\right\},
\end{aligned}
$$

where we define the Banach spaces

$$
\begin{aligned}
C_{v}^{0}\left(\mathbb{R}^{-}\right) & =\left\{f \in C^{0}\left(\mathbb{R}^{-}\right) ;|f(y)|(1+|y|)^{v}<\infty\right\}, \quad v>0, \\
C_{v}^{1}\left(\mathbb{R}^{-}\right) & =\left\{f \in C_{v}^{0}\left(\mathbb{R}^{-}\right), f^{\prime} \in C_{v}^{0}\left(\mathbb{R}^{-}\right)\right\}, \\
C_{\lim , v}^{0}\left(\mathbb{R}^{-}\right) & =\left\{f \in C^{0}\left(\mathbb{R}^{-}\right) ; \exists l \in \mathbb{R},|f(y)-l|(1+|y|)^{v}<\infty\right\}, \\
C_{\lim , v}^{1}\left(\mathbb{R}^{-}\right) & =\left\{f \in C_{\lim , v}^{0}\left(\mathbb{R}^{-}\right) ; f^{\prime} \in C_{v}^{0}\left(\mathbb{R}^{-}\right)\right\},
\end{aligned}
$$


and we take for $\left(a, b, f_{1}, g_{1}, f_{2}, g_{2}\right)^{t}=V \in \mathbb{H}$, the norm

$$
|V|_{\mathbb{H}}=|a|+|b|+\left\|f_{1}\right\|_{1, \infty}+\left\|g_{1}\right\|_{1, \infty}^{\lim }+\left\|f_{2}\right\|_{\infty}+\left\|g_{2}\right\|_{\infty},
$$

with

$$
\begin{aligned}
& \|f\|_{\nu, \infty} \stackrel{\text { def }}{=} \sup _{y \in \mathbb{R}^{-}}\left(|f(y)|(1+|y|)^{\nu}\right), \quad\|f\|_{\infty} \stackrel{\text { def }}{=} \sup _{y}|f(y)|, \\
& \|g\|_{\nu, \infty}^{\lim } \stackrel{\text { def }}{=} \sup _{y \in \mathbb{R}^{-}}|g(y)|+\sup _{y \in \mathbb{R}^{-}}\left(|g(y)-l|(1+|y|)^{\nu}\right) .
\end{aligned}
$$

The definition of the norm in $\mathbb{D}$ is similar, in adding the norms of $f_{j}^{\prime}$ and $g_{j}^{\prime}$. The system (4) is reversible, i.e. $F$ anticommutes with the symmetry $S$ which reads

$$
S U=\left(\beta_{10}, \beta_{21},-\alpha_{1}, \beta_{1},-\alpha_{2}, \beta_{2}\right) .
$$

Notice that the interface and free surface, expressed in the new coordinates satisfy

$$
\frac{d Z_{I}}{d x}=\mathrm{e}^{-\beta_{10}} \sin \alpha_{10}, \quad \frac{d Z}{d x}=\mathrm{e}^{\beta_{20}-\beta_{10}-\beta_{21}} \sin \alpha_{21} .
$$

For looking for homoclinic connections, we work with the rescaled system

$$
\frac{d \underline{U}}{d \underline{x}}=\underline{F}(\varepsilon ; \underline{U}),
$$

where

$$
\varepsilon U=\underline{U}, \quad \varepsilon x=\underline{x}, \quad \varepsilon y=\underline{y} \quad \text { for } y \in] 0,-\infty[.
$$

The differential at the origin $\underline{L}_{\varepsilon}=D \underline{F}(\varepsilon, 0)$ admits for eigenvalues $0, \pm i \lambda_{\varepsilon} / \varepsilon$ with the corresponding eigenvectors given by

$$
\begin{aligned}
& \xi_{0}=(0,1,0,0,0,1), \quad \xi_{1}=(1,0,0,1,0,0), \quad L_{\varepsilon} \xi_{0}=L_{\varepsilon} \xi_{1}=0, \\
& S \xi_{0}=\xi_{0}, \quad S \xi_{1}=\xi_{1}, \\
& \underline{\zeta}_{\varepsilon}=\left(1, \mathrm{e}^{\lambda_{\varepsilon}},-i \mathrm{e}^{\lambda_{\varepsilon} \underline{y} / \varepsilon}, \mathrm{e}^{\lambda_{\varepsilon} \underline{y} / \varepsilon},-i \mathrm{e}^{\lambda_{\varepsilon} y}, \mathrm{e}^{\lambda_{\varepsilon} y}\right), \quad \underline{L}_{\varepsilon} \underline{\zeta}_{\varepsilon}=\frac{i \lambda}{\varepsilon} \underline{\zeta}_{\varepsilon}, \quad \bar{\zeta}=S \underline{\zeta}_{\varepsilon} .
\end{aligned}
$$

Let us define some associated linear forms and projections: for $V=\left(a, b, f_{1}, g_{1}\right.$, $\left.f_{2}, g_{2}\right) \in \mathbb{H}$ we set

$$
\begin{array}{ll}
p_{0}^{*}(V)=g_{21}=g_{2}(1), & p_{1}^{*}(V)=a, \quad \underline{p}_{\varepsilon}(V)=\zeta_{\varepsilon}^{*}(V) \underline{\zeta}_{\varepsilon}+\bar{\zeta}_{\varepsilon}^{*}(V) \bar{\zeta}_{\varepsilon}, \\
\pi_{\varepsilon}(V)=\mathrm{Id}-\underline{p}_{\varepsilon}(V), & \underline{\pi}_{\varepsilon}=\pi_{\varepsilon}-\xi_{0} p_{0}^{*} \pi_{\varepsilon}
\end{array}
$$

with

$$
\begin{aligned}
\zeta_{\varepsilon}^{*}(V)= & d\left\{a-\rho g_{20}+\rho \mathrm{e}^{\lambda_{\varepsilon}} b+\rho \lambda_{\varepsilon} \int_{0}^{1}\left[i f_{2}(y)-g_{2}(y)\right] \mathrm{e}^{\lambda_{\varepsilon} y} d y\right. \\
& \left.+\lambda \int_{-\infty}^{0}\left[i f_{1}(\varepsilon \tau)-g_{1}(\varepsilon \tau)\right] \mathrm{e}^{\lambda \tau} d \tau\right\} .
\end{aligned}
$$


These projections satisfies

$$
\begin{array}{ll}
p_{0}^{*}\left(\xi_{0}\right)=1, & p_{0}^{*}\left(\xi_{1}\right)=0, \quad p_{0}^{*}\left(\underline{\zeta}_{\varepsilon}\right)=p_{0}^{*}\left(\underline{\zeta}_{\varepsilon}\right)=\mathrm{e}^{\lambda_{\varepsilon}}, \\
p_{1}^{*}\left(\xi_{0}\right)=0, \quad p_{1}^{*}\left(\xi_{1}\right)=1, \quad p_{1}^{*}\left(\underline{\zeta}_{\varepsilon}\right)=p_{1}^{*}\left(\underline{\zeta}_{\varepsilon}\right)=1, \\
\underline{p}_{\varepsilon}\left(\xi_{0}\right)=\underline{p}_{\varepsilon}\left(\xi_{1}\right)=0, \quad \underline{p}_{\varepsilon}\left(\underline{\zeta}_{\varepsilon}\right)=\underline{\zeta}_{\varepsilon}, \quad \underline{p}_{\varepsilon}\left(\underline{\zeta}_{\varepsilon}\right)=\underline{\zeta}_{\varepsilon} .
\end{array}
$$

\subsection{Nonlocal normal form formulation}

As already explained our aim is to look for generalized solitary waves with polynomial decay at infinity. This waves appear as homoclinic connections of the infinite dimensional dynamical system (4) or equivalently as homoclinic connections of its rescaled form (7). For describing this expected decay rate in $\underline{x}$ of the solutions we introduce the following Banach spaces:

Definition 2. - Let $\mathbb{E}$ be a Banach space and $\alpha$ be in $] 0,1[$. Let us define

$$
\begin{aligned}
B_{p}^{\alpha}(\mathbb{E}) & =\left\{f \in C^{\alpha}(\mathbb{E}) ;\|f\|_{B_{p}^{\alpha}(\mathbb{E})}<\infty\right\}, \\
B_{p}^{1, \alpha}(\mathbb{E}) & =\left\{f \in B_{p}^{\alpha}(\mathbb{E}) ; \frac{d f}{d \underline{x}} \in B_{p}^{\alpha}(\mathbb{E})\right\},
\end{aligned}
$$

with

$$
\|f\|_{B_{p}^{\alpha}(\mathbb{E})}=\sup _{x \in \mathbb{R}}\left(1+|x|^{p}\right)|f(x)|_{\mathbb{E}}+\sup _{x \in \mathbb{R}, \delta \in]-1,1[}\left(1+|x|^{p}\right) \frac{|f(x+\delta)-f(x)|_{\mathbb{E}}}{|\delta|^{\alpha}} .
$$

We also introduce the spaces $B_{\mathbb{H}, w}^{\alpha}, B_{\mathbb{H}, w}^{1, \alpha}$ and $B_{\mathbb{D}, w}^{\alpha}$ defined by

$$
\begin{aligned}
B_{\mathbb{H}, w}^{\alpha}=\{ & \left\{=\left(a, b, f_{1}, g_{1}, f_{2}, g_{2}\right) ; V(x) \in \mathbb{H},\right. \\
& \left.(a, b) \in B_{2}^{\alpha}\left(\mathbb{R}^{2}\right),\left(f_{1}, g_{1}\right) \in\left(B_{w}^{-}\right)^{2},\left(f_{2}, g_{2}\right) \in\left(B_{w}^{+}\right)^{2}\right\}, \\
B_{\mathbb{D}, w}^{\alpha}=\{ & \left(U=\left(\beta_{10}, \beta_{21}, \alpha_{1}, \beta_{1}, \alpha_{2}, \beta_{2}\right) ; U(x) \in \mathbb{D},\right. \\
& \left.\left(\alpha_{1}, \beta_{1}\right) \in\left(B_{w}^{-}\right)^{2},\left(\alpha_{2}, \beta_{2}\right) \in\left(B_{w}^{1,+}\right)^{2}\right\}, \\
B_{\mathbb{H}, w}^{1, \alpha}= & \left\{V \in B_{\mathbb{H}, w}^{\alpha} ; \frac{d V}{d \underline{x}} \in B_{\mathbb{H}, w}^{\alpha}\right\},
\end{aligned}
$$

where

$$
\begin{aligned}
& B_{w}^{-}=\left\{f(x, y) ;(x, y) \in \mathbb{R} \times \mathbb{R}^{-}, f \text { is } C^{\alpha} \text { in } x, C^{0} \text { in } y,\|f\|_{B_{w}^{-}}<\infty\right\}, \\
& B_{w}^{+}=B_{2}^{\alpha}\left[C^{0}(0,1)\right], \quad B_{w}^{1,+}=B_{2}^{\alpha}\left[C^{1}(0,1)\right] \\
& \|f\|_{B_{w}^{-}}=\sup _{x \in \mathbb{R}, y<0} \frac{\left(1+|x|^{2}+|y|^{2}\right)}{1+|y|}|f(x, y)| \\
& \quad+\sup _{x \in \mathbb{R}, y<0,|\delta| \leqslant 1} \frac{\left(1+|x|^{2}+|y|^{2}\right)}{1+|y|} \frac{|f(x+\delta, y)-f(x, y)|}{|\delta|^{\alpha}}
\end{aligned}
$$

For looking for homoclinic connections we first use the following "Nonlocal Normal Form Lemma" proved in [7] (see Lemma 18) which ensures that the full Euler equations 
(7) can be reduced via an appropriate change of coordinates to a Benjamin-Ono equation coupled with the equation of a linear, high frequency oscillator.

LEMma 3 (Nonlocal Normal Form Lemma). - There exist $\delta_{1}$ and an analytic change of coordinates close to identity

$$
\underline{U}=\Upsilon(A, \bar{A}, u, Y) \quad \text { with }(A(\underline{x}), \bar{A}(\underline{x}), u(\underline{x}), Y(\underline{x})) \in \mathbb{C}^{2} \times \mathbb{R} \times \underline{\pi}_{\varepsilon} \mathbb{D} \text { for } \underline{x} \in \mathbb{R}
$$

such that for any $\left.\left.\delta_{0}, \varepsilon \in\right] 0, \varepsilon_{0}\left(\delta_{0}\right)\right]$, and any function $x \mapsto(A(\underline{x}), \bar{A}(\underline{x}), u(\underline{x}), Y(\underline{x}))$ lying in

$$
E^{\alpha}:=\left(C^{\alpha}(\mathbb{R}, \mathbb{C})\right)^{2} \times B_{2}^{1, \alpha}(\mathbb{R}) \times B_{\underline{\pi}_{\varepsilon}}^{\alpha} \mathbb{D}, w
$$

and satisfying $|A|<\delta_{1},|u|+\varepsilon|Y|_{\underline{\pi}_{\varepsilon} \mathbb{D}}<\delta_{0}$, (7) is equivalent to

$$
\begin{aligned}
& \frac{d A}{d \underline{x}}-i A\left(\frac{\lambda_{\varepsilon}}{\varepsilon}+\gamma_{1}\left(u, \varepsilon p_{1}^{*}(Y),|A|^{2}, \varepsilon\right)\right)=R_{\varepsilon, A}(A, \bar{A}, u, Y), \\
& \rho \mathcal{H}\left[\frac{d u}{d \underline{x}}\right]+u+\frac{3}{2} u^{2}=\mathcal{R}_{\varepsilon, u}[A, \bar{A}, u, Y]+R_{\varepsilon, u}\left(|A|^{2}\right)+c_{0}, \\
& Y-\mathcal{T}_{0}[u]=\mathcal{R}_{\varepsilon, Y}[A, \bar{A}, u, Y],
\end{aligned}
$$

with

$$
\begin{aligned}
& \mathcal{R}_{\varepsilon, Y}[A, \bar{A}, u, Y]=\mathcal{T}_{1}\left[T_{u}(A, \bar{A}, u, Y)\right]+\mathcal{T}_{2}\left[T_{Y}(A, \bar{A}, u, Y)\right], \\
& \mathcal{R}_{\varepsilon, u}[A, \bar{A}, u, Y]=\frac{\varepsilon}{\lambda} \mathcal{H}\left[\frac{d u}{d \underline{x}}\right]+\mathcal{C}_{\varepsilon}^{(1)}\left[T_{u}\right]+\mathcal{C}_{\varepsilon}^{(2)}\left[T_{Y}\right]+B_{u}(A, \bar{A}, u, Y),
\end{aligned}
$$

where $c_{0}$ is an arbitrary constant; $R_{\varepsilon, u}$ is a real analytic function; $R_{\varepsilon, A}, T_{u}, T_{Y}, B_{u}$ are nonlinear analytic function of $(A, \bar{A}, u, Y)$ and $\mathcal{T}_{0}, \mathcal{T}_{1}, \mathcal{T}_{2}, \mathcal{C}_{\varepsilon}^{(1)}, \mathcal{C}_{\varepsilon}^{(2)}$, are linear nonlocal operators, $\mathcal{H}$ being the Hilbert transform. Moreover $\gamma_{1}$ is explicitly given by

$$
\gamma_{1}\left(u, v,|A|^{2}, \varepsilon\right)=\sum_{1 \leqslant n+m+2 p \leqslant r} \gamma_{n m p r} u^{n} v^{m}|A|^{2 p} \varepsilon^{r-1} \in \mathbb{R}
$$

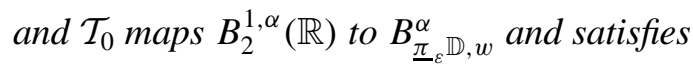

$$
\left\|\mathcal{T}_{0}[u]\right\|_{B_{\mathbb{D}, w}^{\alpha}} \leqslant M\|u\|_{B_{2}^{1, \alpha}(\mathbb{R})} .
$$

For any function $x \mapsto(A(\underline{x}), \bar{A}(\underline{x}), u(\underline{x}), Y(\underline{x}))$ lying in $\left(C^{\alpha}(\mathbb{R}, \mathbb{C})\right)^{2} \times B_{2}^{1, \alpha}(\mathbb{R}) \times$

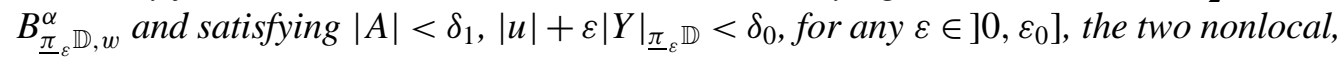
nonlinear perturbations terms $\mathcal{R}_{\varepsilon, u}[A, \bar{A}, u, Y]$ and $\mathcal{R}_{\varepsilon, Y}[A, \bar{A}, u, Y]$ lie respectively in

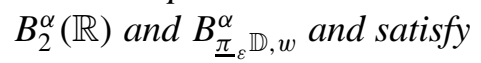

$$
\begin{aligned}
& \left\|\mathcal{R}_{\varepsilon, u}[A, \bar{A}, u, Y]\right\|_{B_{2}^{\alpha}(\mathbb{R})} \leqslant M \varepsilon\|Y\|_{B_{\underline{\pi}_{\varepsilon}, \mathbb{D}, w}^{\alpha}}\left(\|u\|_{B_{2}^{1, \alpha}(\mathbb{R})}+\varepsilon\|Y\|_{B_{\mathbb{\pi}_{\varepsilon} \mathbb{D}, w}^{\alpha}}+\|A\|_{C^{\alpha}}\right) \\
& +M \varepsilon\|u\|_{B_{2}^{1, \alpha}(\mathbb{R})}\left(1+\|A\|_{C^{\alpha}}\|A\|_{C^{0}}\right), \\
& \left\|\mathcal{R}_{\varepsilon, Y}[A, \bar{A}, u, Y]\right\|_{B_{\underline{\underline{x}}_{\varepsilon}}^{\alpha} \mathbb{D}, w} \leqslant M \varepsilon\|Y\|_{B_{\underline{\pi}_{\varepsilon} \mathbb{D}, w}^{\alpha}}\left(\|u\|_{B_{2}^{1, \alpha}(\mathbb{R})}+\varepsilon\|Y\|_{B_{\underline{\pi}_{\varepsilon}}^{\alpha} \mathbb{\mathbb { D } , w}}+\|A\|_{C^{\alpha}}\right),
\end{aligned}
$$




$$
\begin{aligned}
& \left\|D \mathcal{R}_{\varepsilon, u}[A, \bar{A}, u, Y]\right\|_{\mathcal{L}\left(E^{\alpha}, B_{2}^{\alpha}(\mathbb{R})\right)} \leqslant M \varepsilon\left(1+\|u\|_{B_{2}^{1, \alpha}(\mathbb{R})}+\|Y\|_{B_{\mathbb{\pi}_{\varepsilon} \mathbb{D}, w}^{\alpha}}+\|A\|_{C^{\alpha}}\right), \\
& \left\|D \mathcal{R}_{\varepsilon, Y}[A, \bar{A}, u, Y]\right\|_{\mathcal{L}\left(E^{\alpha}, B_{\underline{\pi}_{\varepsilon} \mathbb{D}, w}^{\alpha}\right)} \leqslant M \varepsilon\left(1+\|u\|_{B_{2}^{1, \alpha}(\mathbb{R})}+\|Y\|_{B_{\underline{\pi}_{\varepsilon} \mathbb{D}, w}^{\alpha}}+\|A\|_{C^{\alpha}}\right) .
\end{aligned}
$$

Finally the local nonlinear perturbation term $R_{\varepsilon, A}$ satisfies

$$
\left|R_{\varepsilon, A}(A, \bar{A}, u, Y)\right| \leqslant M \varepsilon|Y|_{\mathbb{D}}\left(|u|+|Y|_{\mathbb{D}}\right)
$$

for $|A|<\delta_{1},|u|+\varepsilon|Y|_{\underline{\pi}_{\varepsilon} \mathbb{D}}<\delta_{0}$, and $\left.\left.\varepsilon \in\right] 0, \varepsilon_{0}\right]$.

This system is still reversible, which now reads

$$
R_{\varepsilon, A}(\bar{A}, A, u, S Y)=-\overline{R_{\varepsilon, A}(A, \bar{A}, u, Y)}
$$

for the local perturbation term and

$$
\begin{aligned}
& \hat{s} \circ \mathcal{R}_{\varepsilon, u}[A, \bar{A}, u, Y]=\mathcal{R}_{\varepsilon, u}[\hat{s} \circ \bar{A}, \hat{s} \circ A, \hat{s} \circ u, \widehat{S} \circ Y], \\
& \widehat{S} \circ \mathcal{R}_{\varepsilon, Y}[A, \bar{A}, u, Y]=\mathcal{R}_{\varepsilon, Y}[\hat{s} \circ \bar{A}, \hat{s} \circ A, \hat{s} \circ u, \widehat{S} \circ Y]
\end{aligned}
$$

for the nonlocal perturbations terms where $(\hat{s} \circ f)(x)=f(-x)$ for real or complex valued functions $f$ and $(\widehat{S} \circ Y)(x)=S Y(-x)$ for function $Y$ mapping $\mathbb{R}$ in $\mathbb{H}$ or $\mathbb{D}$.

Observe that the full system (9) admits a family of reversible periodic orbits of arbitrary small size explicitly given by

$$
\mathrm{p}_{A_{0}, \varepsilon}(\underline{x})=\left(A_{0} \mathrm{e}^{i s(\underline{x})}, A_{0} \mathrm{e}^{-i s(\underline{x})}, 0,0\right)
$$

with

$$
s(\underline{x})=\left[\frac{\lambda}{\varepsilon}+\gamma_{1}\left(0,0, A_{0}^{2}, \varepsilon\right)\right] \underline{x} \text { and } A_{0} \in \mathbb{R},
$$

whereas the truncated system corresponding to $R_{\varepsilon, A}=\mathcal{R}_{\varepsilon, u}=\mathcal{R}_{\varepsilon, Y} \equiv 0$ is partially decoupled and admits for $c_{0}=-R_{\varepsilon, u}\left(\left|A_{0}\right|^{2}\right)$ a family of homoclinic connections to the previously found periodic orbits $\mathrm{p}_{A_{0}, \varepsilon}$ given by

$$
\mathrm{h}_{A_{0}, \varphi, \varepsilon}=\left(A_{\varphi}^{h}, \overline{A_{\varphi}^{h}}, u_{0}^{h}, Y_{0}^{h}\right), \quad \mathrm{h}_{A_{0}, \varphi, \varepsilon}(\underline{x}) \underset{\underline{x \rightarrow \pm \infty}}{\longrightarrow} \mathrm{p}_{A_{0}, \varepsilon}\left(\underline{x} \pm \psi_{\infty}+\varphi\right)
$$

with

$$
\begin{aligned}
& u_{0}^{h}(\underline{x})=\frac{-4 \rho^{2}}{3\left(\rho^{2}+\underline{x}^{2}\right)} \in B_{2}^{1, \alpha}(\mathbb{R}) \quad \text { is even in } \underline{x}, \\
& Y_{0}^{h}=\mathcal{T}_{0}\left[u_{0}^{h}\right] \in B_{\underline{\underline{\pi}}_{\varepsilon} \mathbb{D}, w}^{\alpha} \quad \text { is reversible, } \\
& \psi_{\infty}=\int_{0}^{+\infty}\left(\gamma_{1}\left(u_{0}^{h}(\tau), \varepsilon p_{1}^{*}\left(Y_{0}^{h}(\tau)\right), A_{0}^{2}, \varepsilon\right)-\gamma_{1}\left(0,0, A_{0}^{2}, \varepsilon\right)\right) d \tau, \\
& A_{\varphi}^{h}(\underline{x})=A_{0} \mathrm{e}^{i \Psi(\underline{x})+i \varphi}
\end{aligned}
$$


and

$$
\Psi(\underline{x})=\left(\frac{\lambda_{\varepsilon}}{\varepsilon}+\gamma_{1}\left(0,0, A_{0}^{2}, \varepsilon\right)\right) \underline{x}+\int_{0}^{\underline{x}} \gamma_{1}\left(u_{0}^{h}(\tau), \varepsilon p_{1}^{*}\left(Y_{0}^{h}(\tau)\right), A_{0}^{2}, \varepsilon\right) d \tau,
$$

where $\phi$ is arbitrary and $u_{0}^{h}$ satisfies the Benjamin-Ono equation (9-b) with $\mathcal{R}_{\varepsilon, u} \equiv 0$.

Among this two parameters family there are two one parameter families of reversible homoclinic connections corresponding to appropriate choices of the phase shift $\varphi$,

$$
\mathrm{h}_{A_{0}, 0, \varepsilon} \text { and } \mathrm{h}_{A_{0}, \pi, \varepsilon},
$$

and a unique homoclinic connection to $0, \mathrm{~h}_{0, \varepsilon}$ given by

$$
\mathrm{h}_{0, \varepsilon}=\left(0,0, u_{0}^{h}, Y_{0}^{h}\right) .
$$

The question is then the persistence of this family of homoclinic connections for the full system (9) seen as a perturbation of the truncated system by the perturbation terms $R_{\varepsilon, A}, \mathcal{R}_{\varepsilon, u}, \mathcal{R}_{\varepsilon, Y}$.

The system (9) clearly shows the competition between the oscillatory dynamics induced by Eq. (9-a) which generates periodic solutions, and the Benjamin-Ono like dynamics induced by Eqs. (9-b), (9-c) which generate homoclinic connections to 0 with polynomial decay. For the truncated system these two dynamics are decoupled, and there is no competition, so there exist homoclinic connections to periodic orbits of arbitrary small size. Such a coexistence of an oscillatory dynamics and a hyperbolic dynamics also occurs for one parameter families of reversible vector fields admitting a $0^{2} i \omega$ resonance at the origin, i.e. vector fields admitting the origin as a fixed point and such that the differential at the origin admits the bifurcation of spectrum described in Fig. 4. For such vector fields it is proved in [10] that there is generically no homoclinic connection to 0 , whereas there are always homoclinic connections to exponentially small periodic orbits.

In [7], the persistence of the homoclinic connections $\mathrm{h}_{A_{0}, 0, \varepsilon}$ is proved provided that the size of the limiting periodic orbits $A_{0}$ satisfies $A_{0} \geqslant \delta \varepsilon^{3 / 2}$. In this paper we improve this result by showing the persistence for $A_{0} \geqslant c(\ell) \mathrm{e}^{-\lambda_{0} \ell / \varepsilon}$ for $0<\ell<\rho$. As for the $0^{2} i \omega$ resonance we expect a generic non existence of homoclinic connections to 0 , however the proof remains to be done in this case.

The competition between the oscillatory dynamics and the Benjamin-Ono like dynamics causes the appearance of oscillations at infinity for the solutions. The size of the oscillations is given by an oscillatory integral of the form

$$
I(\varepsilon)=\int_{-\infty}^{+\infty} g(\mathrm{U}(s), \varepsilon) \mathrm{e}^{\frac{i \lambda_{0} s}{\varepsilon}} d s
$$

where $g$ is explicitly known whereas $\mathrm{U}=(A, \bar{A}, u, Y)$ is a solution of (9). In the next section we introduce appropriate algebras of holomorphic functions which allow to obtain exponentially small upper bounds of such oscillatory integrals. 


\section{Exponentially small estimates of oscillatory integrals and complexification}

Lemma 4 (Exponential Lemma). - Let $\ell, \omega$ be two positive real numbers and let $p>1$. We denote by $\mathfrak{B}_{\ell}$ the strip in the complex field: $\mathfrak{B}_{\ell}=\{z \in \mathbb{C} /|\operatorname{I} m(z)|<\ell\}$ and by $\mathcal{H}_{\ell, p}$ the set of functions $\left.\left.f: \mathfrak{B}_{\ell} \times\right] 0,1\right] \rightarrow \mathbb{C}$ satisfying

(a) $\xi \mapsto f(\xi, \varepsilon)$ is holomorphic in $\mathfrak{B}_{\ell}$,

(b) $\|f\|_{\mathcal{H}_{\ell, p}}:=\sup _{\varepsilon \in] 0,1], z \in \mathfrak{B}_{\ell}}\left(|f(z, \varepsilon)|\left(1+|z|^{p}\right)\right)<+\infty$.

Then for every $f \in \mathcal{H}_{\ell, p}$ and $\left.\left.\varepsilon \in\right] 0,1\right], I^{ \pm}(f, \varepsilon)=\int_{-\infty}^{+\infty} f(t, \varepsilon) \mathrm{e}^{ \pm i \omega t / \varepsilon} d t$ satisfies

$$
\left|I^{ \pm}(f, \varepsilon)\right| \leqslant c_{p}\|f\|_{\mathcal{H}_{\ell, p}} \mathrm{e}^{-\omega \ell / \varepsilon} \quad \text { with } c_{p}=2 \int_{0}^{+\infty} \frac{1}{1+t^{p}} .
$$

Proof. - We only do the proof for $I^{+}$. For $I^{-}$, perform the change of time $t^{\prime}=-t$ in the integral. So, let $f \in \mathcal{H}_{\ell, p}, \varepsilon, \ell^{\prime}<\ell$ be fixed. Since $f$ is holomorphic in $\mathfrak{B}_{\ell}$, the integral of $f \mathrm{e}^{i \omega t / \varepsilon}$ along the path $\Gamma_{1}$ given in Fig. 6, is equal to zero. Pushing $R$ to $+\infty$, we get

$$
I^{+}(f, \varepsilon)=\int_{-\infty}^{+\infty} f\left(i \ell^{\prime}+t, \varepsilon\right) \mathrm{e}^{i \omega\left(i \ell^{\prime}+t\right) / \varepsilon} d t .
$$

The estimate then follows, where the exponential comes from the oscillating term computed on the line $\operatorname{Im}(z)=\ell^{\prime}$.

Lemma 4 gives a very efficient way to obtain exponential upper bounds because the membership to $\mathcal{H}_{\ell, p}$ is stable by addition, multiplication, and "composition", which can be summed up as follows

LEMMA 5. $-\mathcal{H}_{\ell, p}$ is an algebra and if $f \in \mathcal{H}_{\ell, p}$ and $g$ is holomorphic in a domain containing the range of $f$ and satisfies $g(0)=0$, then $g \circ f \in \mathcal{H}_{\ell, p}$.

For using the exponential Lemma 4 to compute an exponentially small upper bound of oscillatory integral of the form (11) we need to complexify the problem and to look for solutions of (9) in spaces of type $\mathcal{H}_{\ell, p}$. For that purpose we introduce the complexified spaces corresponding to the real ones given in Definition 2.

Definition 6. - Let $\mathbb{E}$ be a real Banach space and $\alpha$ be in $] 0,1[$. Denote by $\widehat{\mathbb{E}}$ the complexified of $\mathbb{E}$, i.e. $\widehat{\mathbb{E}}=\mathbb{E}+i \mathbb{E}$.

For any $\ell>0$, any function $f: \mathfrak{B}_{\ell} \rightarrow \widehat{\mathbb{E}}$ and any $\left.\eta \in\right]-\ell, \ell\left[\right.$, we denote by $\left.f\right|_{\eta}: \mathbb{R} \rightarrow$ $\widehat{\mathbb{E}},\left.f\right|_{\eta}=f(\cdot+i \eta)$.

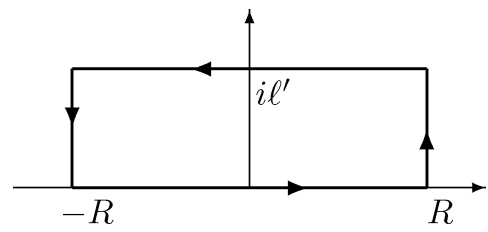

Fig. 6. Path $\Gamma_{1}$. 
Then, let us define the Banach spaces

$$
\begin{aligned}
& H_{\ell, p}^{\alpha}(\widehat{\mathbb{E}})=\left\{f: \mathfrak{B}_{\ell} \rightarrow \widehat{\mathbb{E}}, \text { holomorphic; } f(\mathbb{R}) \subset \mathbb{E} ; \forall \eta \in\right]-\ell, \ell\left[,\left.f\right|_{\eta} \in B_{p}^{\alpha}(\widehat{\mathbb{E}}) ;\right.\left.\|f\|_{H_{\ell, p}^{\alpha}(\widehat{\mathbb{E}})}=\sup _{\eta \in]-\ell, \ell[}\left\|\left.f\right|_{\eta}\right\|_{B_{p}^{\alpha}(\widehat{\mathbb{E}})}<\infty\right\}, \\
& H_{\ell, p}^{1, \alpha}(\widehat{\mathbb{E}})=\left\{f \in H_{\ell, p}^{\alpha}(\widehat{\mathbb{E}}) ; \frac{d f}{d z} \in H_{\ell, p}^{\alpha}(\widehat{\mathbb{E}})\right\}, \\
& C^{\alpha}\left(\mathfrak{B}_{\ell}, \widehat{\mathbb{E}}\right)=\left\{f: \mathfrak{B}_{\ell} \rightarrow \widehat{\mathbb{E}} ; f(\mathbb{R}) \subset \mathbb{E} ; \forall \eta \in\right]-\ell, \ell\left[,\left.f\right|_{\eta} \in C^{\alpha}(\mathbb{R}, \widehat{\mathbb{E}}) ;\right. \\
&\left.\|f\|_{C^{\alpha}\left(\mathfrak{B}_{\ell}, \widehat{\mathbb{E}}\right)}=\sup _{\eta \in]-\ell, \ell[}\left\|\left.f\right|_{\eta}\right\|_{C^{\alpha}(\mathbb{R}, \widehat{\mathbb{E}})}<\infty\right\} .
\end{aligned}
$$

To shorten notations, we denote

$$
H_{\ell, p}^{\alpha}:=H_{\ell, p}^{\alpha}(\mathbb{C}), \quad \text { and } \quad H_{\ell, p}^{1, \alpha}:=H_{\ell, p}^{1, \alpha}(\mathbb{C})
$$

We also introduce the Banach spaces $H_{\mathbb{H}, w}^{\alpha}, H_{\mathbb{H}, w}^{1, \alpha}$ and $H_{\mathbb{D}, w}^{\alpha}$ defined by

$$
\begin{aligned}
H_{\mathbb{H}, w}^{\alpha}= & \left\{V: \mathfrak{B}_{\ell} \rightarrow \widehat{\mathbb{H}}, \text { holomorphic, } V(\mathbb{R}) \subset \mathbb{H},\right. \\
& \forall \eta \in]-\ell, \ell\left[,\left.\quad V\right|_{\eta} \in \widehat{B_{\mathbb{H}, w}^{\alpha}}:=B_{\mathbb{H}, w}^{\alpha}+i B_{\mathbb{H}, w}^{\alpha} ;\right. \\
& \left.\|V\|_{H_{\mathbb{H}, w}^{\alpha}}^{\alpha}=\sup _{\eta \in]-\ell, \ell[}\left\|\left.V\right|_{\eta}\right\|_{\widehat{B_{\mathbb{H}, w}^{\alpha}}}^{\alpha}<\infty\right\}, \\
H_{\mathbb{D}, w}^{\alpha}= & \left\{V: \mathfrak{B}_{\ell} \rightarrow \widehat{\mathbb{D}}, \text { holomorphic, } V(\mathbb{R}) \subset \mathbb{D},\right. \\
& \forall \eta \in]-\ell, \ell\left[,\left.V\right|_{\eta} \in \widehat{B_{\mathbb{D}, w}^{\alpha}}:=B_{\mathbb{D}, w}^{\alpha}+i B_{\mathbb{D}, w}^{\alpha} ;\right. \\
& \left.\|V\|_{H_{\mathbb{D}, w}^{\alpha}}^{\alpha}:=\sup _{\eta \in]-\ell, \ell[}\left\|\left.V\right|_{\eta}\right\|_{B_{\mathbb{D}, w}^{\alpha}}^{\alpha}<\infty\right\}, \\
H_{\mathbb{H}, w}^{1, \alpha}=\{ & \left\{V \in H_{\mathbb{H}, w}^{\alpha} ; \frac{d V}{d z} \in H_{\mathbb{H}, w}^{\alpha}\right\} .
\end{aligned}
$$

Observe that $H_{\ell, p}^{\alpha}(\widehat{\mathbb{E}})$ is continuously embedded in $C_{b}^{0}\left(\mathfrak{B}_{\ell}, \widehat{\mathbb{E}}\right)$ and that $H_{\mathbb{H}, w}^{\alpha}, H_{\mathbb{D}, w}^{\alpha}$ are respectively continuously embedded in $C_{b}^{0}\left(\mathfrak{B}_{\ell}, \widehat{\mathbb{H}}\right)$ and $C_{b}^{0}\left(\mathfrak{B}_{\ell}, \widehat{\mathbb{D}}\right)$ where $C_{b}^{0}(\Omega, E)$ is the set of the functions which are continuous and bounded from $\Omega$ to $E$.

The different local and nonlocal operators involved in Eq. (9) can be extended to "complex functions" $\mathrm{U}=(A, \widetilde{A}, u, Y)$ lying in

$$
\begin{aligned}
\mathrm{BE}_{\ell}^{\alpha}\left(\delta_{0}, \delta_{1}\right):=\{ & \left\{\mathrm{U} \in \mathrm{E}_{\ell}^{\alpha} / \forall z \in \mathfrak{B}_{\ell},|\widetilde{A}(z)|<\delta_{1},|A(z)|<\delta_{1},\right. \\
& \left.|u(z)|+\varepsilon|Y(z)|_{\widehat{\pi_{\varepsilon} \mathbb{D}}}<\delta_{0}\right\},
\end{aligned}
$$

where

$$
\mathrm{E}_{\ell}^{\alpha}:=\left(C^{\alpha}\left(\mathfrak{B}_{\ell}, \mathbb{C}\right)\right)^{2} \times H_{\ell, 2}^{1, \alpha} \times H_{\underline{\pi}_{\mathbb{E}} \mathbb{D}, w}^{\alpha} \cap\{\mathrm{U} / \widetilde{A}(\underline{x})=\bar{A}(\underline{x}), \text { for } \underline{x} \in \mathbb{R}\}
$$

is normed with

$$
\|U\|_{E_{\ell}^{\alpha}}=\|A\|_{C^{\alpha}}+\|\widetilde{A}\|_{C^{\alpha}}+\|u\|_{H_{\ell, 2}^{1, \alpha}}+\|Y\|_{H_{\tilde{\pi}_{\varepsilon}}^{\alpha} \mathbb{\mathbb { N }}, w} .
$$


Indeed they appear as finite sum of analytic functions of $(A, \bar{A}, u, Y) \in \mathbb{C}^{2} \times \mathbb{R} \times \underline{\pi}_{\varepsilon} \mathbb{D}$ which naturally extend to analytic functions of $(A, \bar{A}, u, Y) \in \mathbb{C}^{2} \times \mathbb{C} \times \underline{\pi}_{\varepsilon} \mathbb{D}$ composed with nonlocal linear operators $\mathcal{T}_{0}, \mathcal{T}_{1}, \mathcal{T}_{2}, \mathcal{C}_{\varepsilon}^{(1)}, \mathcal{C}_{\varepsilon}^{(2)}$ (see (9)) of the form

$$
\mathcal{K}: f \mapsto \mathcal{K}[f]
$$

with

$$
\mathcal{K}[f](\underline{x}):=\text { p.v. } \int_{-\infty}^{+\infty} K(s) \cdot f(\underline{x}-s) d s,
$$

where $f$ is any function from $\mathbb{R}$ to $\mathbb{E}$ and $K(t)$ is a linear bounded operator in $\mathbb{E}$. For instance, the Hilbert transform reads

$$
\mathcal{H}[u](\underline{x}):=\text { p.v. } \int_{-\infty}^{+\infty} \frac{1}{s} \cdot u(\underline{x}-s) d s .
$$

Such operators can readily be extended to a function $f: \mathfrak{B}_{\ell} \rightarrow \widehat{\mathbb{E}}$ with the same integral formula where $\underline{x}$ lies in $\mathfrak{B}_{\ell}$ instead of $\mathbb{R}$. Moreover we have

LEMmA 7. - Let $\mathbb{E}$ be a real Banach space and $\mathcal{L}(\mathbb{E})$ be the space of bounded linear operators in $\mathbb{E}$. Assume that $K: \mathbb{R} \backslash\{0\} \rightarrow \mathcal{L}(\mathbb{E})$ is $C^{1}$ such that

(i) $\|K(s)\|_{\mathcal{L}(\mathbb{E})} \leqslant C_{0} /|s|,\left\|K^{\prime}(s)\right\|_{\mathcal{L}(\mathbb{E})} \leqslant C_{0} /|s|^{2}$ for $|s| \leqslant 1$,

(ii) $\|K(s)\|_{\mathcal{L}(\mathbb{E})} \leqslant C_{1} /|s|^{2}$ for $|s| \geqslant 1$, and p.v. $\int_{-1}^{1} K(s) d s \in \mathcal{L}(\mathbb{E})$.

Then, the linear map $\mathcal{K}$ defined by

$$
f \mapsto \mathcal{K}[f]=\text { p.v. } \int_{\mathbb{R}} K(s) . f(\cdot-s) d s
$$

is bounded from $H_{\ell, 2}^{\alpha}(\mathbb{E})$ into itself.

Proof. - First observe that

$$
\mathcal{K}[f](z)=\int_{-\infty}^{+\infty} K(s) \cdot(f(z-s)-f(z)) d s+\left(\text { p.v. } \int_{-\infty}^{+\infty} K(s) d s\right) \cdot f(z),
$$

where the first integral is a classical convergent integral without "principal value". This formula combined with Lebegues's theorem ensures that $\mathcal{K}[f]$ is holomorphic in $\mathfrak{B}_{\ell}$. Moreover it is proved in [7] (see Lemma 30) that $\mathcal{K}$ is a bounded linear operator from $B_{2}^{\alpha}(\widehat{\mathbb{E}})$ to itself. Hence, since $\mathcal{K}\left[\left.f\right|_{\eta}\right]=\left.(\mathcal{K}[f])\right|_{\eta}, \mathcal{K}$ is a bounded linear operator from $H_{\ell, 2}^{\alpha}(\widehat{\mathbb{E}})$ to itself.

So, to look for solutions of (9) which admit an holomorphic continuation in $\mathrm{E}_{\ell}^{\alpha}$, we study the "complexified equation"

$$
\frac{d A}{d z}-i A\left(\frac{\lambda_{\varepsilon}}{\varepsilon}+\gamma_{1}\left(u, \varepsilon p_{1}^{*}(Y), A \widetilde{A}, \varepsilon\right)\right)=R_{\varepsilon, A}(A, \widetilde{A}, u, Y),
$$




$$
\begin{aligned}
& \frac{d \widetilde{A}}{d z}+i \widetilde{A}\left(\frac{\lambda_{\varepsilon}}{\varepsilon}+\gamma_{1}\left(u, \varepsilon p_{1}^{*}(Y), A \widetilde{A}, \varepsilon\right)\right)=R_{\varepsilon, \tilde{A}}(A, \widetilde{A}, u, Y), \\
& \rho \mathcal{H}\left[\frac{d u}{d z}\right]+u+\frac{3}{2} u^{2}=\mathcal{R}_{\varepsilon, u}[A, \widetilde{A}, u, Y]+R_{\varepsilon, u}(A \widetilde{A})+c_{0}, \\
& Y-\mathcal{T}_{0}[u]=\mathcal{R}_{\varepsilon, Y}[A, \widetilde{A}, u, Y],
\end{aligned}
$$

where $R_{\varepsilon, \tilde{A}}$ is an analytic function of $(A, \widetilde{A}, u, Y)$ given by

$$
R_{\varepsilon, \tilde{A}}(A, \widetilde{A}, u, Y)=\overline{R_{\varepsilon, A}(\overline{\widetilde{A}}, \bar{A}, \bar{u}, \bar{Y})} .
$$

Remark 8. - Observe that for any solution $U$ of (12) lying in $E_{\ell}^{\alpha}$, the restriction of $U$ to $\mathbb{R}$ is a solution of (9) lying in $\left(C^{\alpha}(\mathbb{R}, \mathbb{C})\right)^{2} \times B_{2}^{1, \alpha}(\mathbb{R}) \times B_{\underline{\pi}_{\varepsilon}}^{\alpha} \mathbb{D}, w$.

The bounds for the linear operators $\mathcal{T}_{0}, \mathcal{T}_{1}, \mathcal{T}_{2}, \mathcal{C}_{\varepsilon}^{(1)}, \mathcal{C}_{\varepsilon}^{(2)}$, which lead to the estimates for $\mathcal{R}_{\varepsilon, u}, \mathcal{R}_{\varepsilon, Y}$ stated for (9), are based on the structure of these non local operators where nonlocal kernels occur. In [7] the estimates of these operators were based on Lemma 30, here extended at Lemma 7 for obtaining the holomorphy in the strip $\mathfrak{B}_{\ell}$. It then

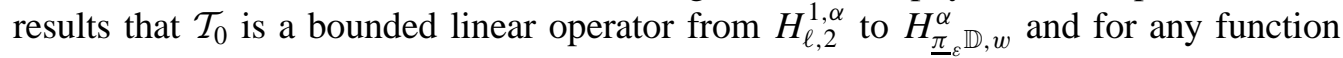
$\mathrm{U}=(A, \widetilde{A}, u, Y)$ lying in $\mathrm{BE}_{\ell}^{\alpha}\left(\delta_{0}, \delta_{1}\right)$ and any $\left.\left.\varepsilon \in\right] 0, \varepsilon_{0}\right]$, the two nonlocal, nonlinear perturbations terms $\mathcal{R}_{\varepsilon, u}[A, \widetilde{A}, u, Y]$ and $\mathcal{R}_{\varepsilon, Y}[A, \widetilde{A}, u, Y]$ lie respectively in $H_{\ell, 2}^{\alpha}$ and

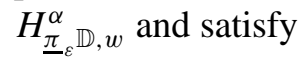

$$
\begin{aligned}
& \left\|\mathcal{R}_{\varepsilon, u}[A, \widetilde{A}, u, Y]\right\|_{H_{\ell, 2}^{\alpha}} \\
& \leqslant M \varepsilon\|Y\|_{H_{\underline{\pi}_{\varepsilon} \mathbb{D}, w}^{\alpha}}\left(\|u\|_{H_{\ell, 2}^{1, \alpha}}+\varepsilon\|Y\|_{H_{\underline{\pi}_{\varepsilon} \mathbb{D}, w}^{\alpha}}+\|A\|_{C^{\alpha}}+\|\widetilde{A}\|_{C^{\alpha}}\right) \\
& +M \varepsilon\|u\|_{H_{\ell, 2}^{1, \alpha}}\left(1+\left(\|A\|_{C^{\alpha}}+\|\widetilde{A}\|_{C^{\alpha}}\right)\left(\|A\|_{C^{0}}+\|\widetilde{A}\|_{C^{0}}\right)\right), \\
& \left\|\mathcal{R}_{\varepsilon, Y}[A, \widetilde{A}, u, Y]\right\|_{H_{\underline{\pi}_{\varepsilon} \mathbb{D}, w}^{\alpha}} \\
& \leqslant M \varepsilon\|Y\|_{H_{\underline{\pi}_{\varepsilon} \mathbb{D}, w}^{\alpha}}\left(\|u\|_{H_{\ell, 2}^{1, \alpha}}+\varepsilon\|Y\|_{H_{\underline{\pi}_{\varepsilon} \mathbb{D}, w}^{\alpha}}+\|A\|_{C^{\alpha}}+\|\widetilde{A}\|_{C^{\alpha}}\right),
\end{aligned}
$$

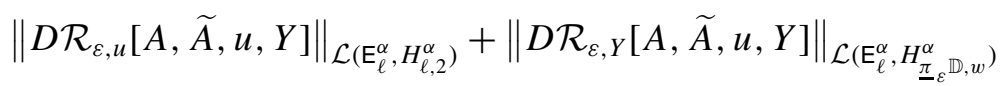

$$
\begin{aligned}
& \leqslant M \varepsilon\left(1+\|u\|_{H_{\ell, 2}^{1, \alpha}}+\|Y\|_{H_{\underline{\pi}_{\varepsilon} \mathbb{D}, w}^{\alpha}}+\|A\|_{C^{\alpha}}+\|\widetilde{A}\|_{C^{\alpha}}\right) .
\end{aligned}
$$

Finally the local nonlinear perturbation terms $R_{\varepsilon, A}, R_{\varepsilon, \tilde{A}}$ satisfy

$$
\left|R_{\varepsilon, A}(A, \widetilde{A}, u, Y)\right|+\left|R_{\varepsilon, \tilde{A}}(A, \widetilde{A}, u, Y)\right| \leqslant M \varepsilon|Y|_{\widehat{\pi_{\varepsilon} \mathbb{D}}}\left(|u|+|Y|_{\widehat{\pi_{\varepsilon} \mathbb{D}}}\right)
$$

for $|A|<\delta_{1},|\widetilde{A}|<\delta_{1},|u|+\varepsilon|Y|_{\widehat{\pi_{\varepsilon} \mathbb{D}}}<\delta_{0}$, and $\left.\left.\varepsilon \in\right] 0, \varepsilon_{0}\right]$.

This system is still reversible, which now reads

$$
R_{\varepsilon, A}(\widetilde{A}, A, u, S Y)=-R_{\varepsilon, \tilde{A}}(A, \widetilde{A}, u, Y)
$$

for the local perturbation term and 


$$
\begin{aligned}
& \hat{s} \circ \mathcal{R}_{\varepsilon, u}[A, \widetilde{A}, u, Y]=\mathcal{R}_{\varepsilon, u}[\hat{s} \circ \widetilde{A}, \hat{s} \circ A, \hat{s} \circ u, \widehat{S} \circ Y], \\
& \widehat{S} \circ \mathcal{R}_{\varepsilon, Y}[A, \widetilde{A}, u, Y]=\mathcal{R}_{\varepsilon, Y}[\hat{s} \circ \widetilde{A}, \hat{s} \circ A, \hat{s} \circ u, \widehat{S} \circ Y]
\end{aligned}
$$

for the nonlocal perturbations terms.

\section{Homoclinic connections to exponentially small periodic orbits}

The purpose of the paper is to prove the existence of generalized solitary waves with exponentially small ripples at infinity. As already explained in Section 2.2, after change of coordinates, the full Euler equations reduces to system (12) and generalized solitary waves appear as homoclinic connections to the periodic orbits $p_{A_{0}, \varepsilon}(\underline{x})$. In this section we prove

THEOREM 9. - For any $\left.\left.\alpha \in] 0, \frac{1}{2}\right], \ell \in\right] 0, \rho\left[\right.$ there exist $c_{\star}, \varepsilon_{\star}$ such that for every $\left.\varepsilon \in] 0, \varepsilon_{\star}\right]$ and every $A_{0} \in\left[c_{\star} \varepsilon \mathrm{e}^{-\ell \lambda_{0} / \varepsilon}, \varepsilon^{1-\alpha} \mathrm{e}^{-\ell \lambda_{0} / \varepsilon}\right]$, system (9) has two reversible homoclinic connections $\mathrm{H}_{A_{0}, \varphi_{j}, \varepsilon}, j=1,2$, of the form

$$
\mathrm{H}_{A_{0}, \varphi_{j}, \varepsilon}(\underline{x})=\mathrm{p}_{A_{0}, \varepsilon}\left(\underline{x}+\varepsilon \varphi_{j} \rho \arctan \frac{\underline{x}}{\rho}\right)+\mathrm{h}_{0, \varepsilon}(\underline{x})+\mathrm{h}_{1, \varepsilon}(\underline{x}),
$$

where $\mathrm{h}_{0, \varepsilon}$ is given by (10) and $\mathrm{h}_{1, \varepsilon}(\underline{x})=\mathcal{O}\left(\varepsilon^{1-\alpha} /(\rho+|\underline{x}|)\right)$.

COROLLARY 10. - There exists $\delta>0$ such that for any $\ell \in] 0, \rho\left[\right.$ there exist $c_{\ell}, \varepsilon_{\ell}$ such that for every $\left.\varepsilon \in] 0, \varepsilon_{\ell}\right]$ and every $A_{0} \in\left[c_{\ell} \varepsilon \mathrm{e}^{-\ell \lambda_{0} / \varepsilon}, \delta\right]$, system (9) has two reversible homoclinic connections $\mathrm{H}_{A_{0}, \varphi_{j}, \varepsilon}, j=1,2$, of the form (16) where $\mathrm{h}_{1, \varepsilon}(\underline{x})=$ $\mathcal{O}(\sqrt{\varepsilon} /(\rho+|\underline{x}|))$.

Remark 11. - Theorem 1 is a direct consequence of the above corollary performing back the change of coordinates given by the Normal Form Lemma 3 and the scaling (8).

Proof of Corollary 10. - We first deduce Corollary 10 from Theorem 9. Looking carefully at the proof of Theorem 9 given in the next subsections, we check that we can obtain a similar theorem which is valid uniformly for any $\left.\left.\ell^{\prime} \in\right] 0, \ell\right]$, i.e. a theorem for which the constants $c_{\star}, \varepsilon_{\star}$ are the same for every $\left.\left.\ell^{\prime} \in\right] 0, \ell\right]$. So grouping together the existence results obtained for $\alpha=\frac{1}{2}$ and each $\left.\left.\ell^{\prime} \in\right] 0, \ell\right]$ and observing that

$$
\bigcup_{0<\ell^{\prime} \leqslant \ell}\left[c_{\star} \varepsilon \mathrm{e}^{-\frac{\ell^{\prime} \lambda_{0}}{\varepsilon}}, \sqrt{\varepsilon} \mathrm{e}^{-\frac{\ell^{\prime} \lambda_{0}}{\varepsilon}}\right]=\left[c_{\star} \varepsilon \mathrm{e}^{-\frac{\ell \lambda_{0}}{\varepsilon}}, \sqrt{\varepsilon}[\right.
$$

we obtain that for any $\ell \in] 0, \rho\left[\right.$ there exist $c_{\ell}, \varepsilon_{\ell}$ such that for every $\left.\left.\varepsilon \in\right] 0, \varepsilon_{\ell}\right]$ and every $A_{0} \in\left[c_{\ell} \varepsilon \mathrm{e}^{-\ell \lambda_{0} / \varepsilon}, \sqrt{\varepsilon}[\right.$, system (9) has two reversible homoclinic connections $\mathrm{H}_{A_{0}, \varphi_{j}, \varepsilon}, j=1,2$, of the form (16) where $\mathrm{h}_{1, \varepsilon}(\underline{x})=\mathcal{O}(\sqrt{\varepsilon} /(\rho+|\underline{x}|))$.

Finally Theorem 22 of [7] gives the existence of two reversible homoclinic connections $\mathrm{H}_{A_{0}, \varphi_{j}, \varepsilon}, j=1,2$, of the form (16) where $\mathrm{h}_{1, \varepsilon}(\underline{x})=\mathcal{O}(\sqrt{\varepsilon} /(\rho+|\underline{x}|))$ for $A_{0} \in\left[c_{0} \varepsilon^{3 / 2}, \delta\right]$ which completes the proof of Corollary 10 .

The rest of this section is devoted to the proof of Theorem 9 . 


\subsection{Complexified shifted system and choice of the parameters}

In the rest of this paper let $\ell \in] 0, \rho[$ and $\alpha \in] 0,1[$ be fixed.

We look for homoclinic connections to the periodic orbit $\mathrm{p}_{A_{0}, \varepsilon}$ under the form

$$
\mathrm{H}_{A_{0}, \varphi, \varepsilon}=\mathrm{p}_{A_{0}, \varepsilon}\left(\underline{x}+\varepsilon \varphi \rho \arctan \frac{\underline{x}}{\rho}\right)+\mathrm{h}_{\varepsilon}(\underline{x}) .
$$

The unknown are $\varphi \in \mathbb{R}$, which is proportional to the phase shift at infinity, and $\mathrm{h}_{\varepsilon}$ which is required to be a reversible homoclinic connection to 0 . As already explained, to obtain exponentially small estimates of $A_{0}$, we need to show that $\mathrm{H}_{A_{0}, \varphi, \varepsilon}$ admits a holomorphic continuation in $\mathfrak{B}_{\ell}$ still denoted by $\mathrm{H}_{A_{0}, \varphi, \varepsilon}$ which lies in $\mathrm{BE}_{\ell}^{\alpha}\left(\delta_{0}, \delta_{1}\right)$ where $\delta_{0}$ can be chosen arbitrarily. For that purpose we look for $\mathrm{H}_{A_{0}, \varphi, \varepsilon}$ as a solution of (12). Moreover, we look for $\mathrm{h}_{\varepsilon}(z)$ with $z \in \mathfrak{B}_{\ell}$ under the form

$$
\mathrm{h}_{\varepsilon}(z)=\left(\left(i q_{1}(z)+q_{2}(z)\right) \mathrm{e}^{i \psi_{\varphi}(z)},\left(-i q_{1}(z)+q_{2}(z)\right) \mathrm{e}^{-i \psi_{\varphi}(z)}, u, Y\right)
$$

with

$$
\psi_{\varphi}(z)=\left[\frac{\lambda_{\varepsilon}}{\varepsilon}+\gamma_{1}\left(0,0, A_{0}^{2}, \varepsilon\right)\right][z+\varepsilon \rho \varphi \arctan (z / \rho)]
$$

with

$$
\arctan z=\int_{[0, z]} \frac{d s}{1+s^{2}}
$$

observing that

$$
\mathrm{T}:\left(q_{1}, q_{2}\right) \mapsto(A, \widetilde{A})=\left(\left(i q_{1}(z)+q_{2}(z)\right) \mathrm{e}^{i \psi_{\varphi}(z)},\left(-i q_{1}(z)+q_{2}(z)\right) \mathrm{e}^{-i \psi_{\varphi}(z)}\right)
$$

is an isomorphism from $\mathbb{C}^{2}$ onto $\mathbb{C}^{2}$.

We need a priori estimates on $\varphi$ and $\mathrm{h}$ to ensure that $\mathrm{H}_{A_{0}, \varphi, \varepsilon}$ lies in $\mathrm{BE}_{\ell}^{\alpha}\left(\delta_{0}, \delta_{1}\right)$. Moreover, we want to prove the existence of homoclinic connection to $\mathrm{p}_{A_{0}, \varepsilon}$ provided that its size $A_{0}$ lies in an interval of the form $\left[c_{\star} \varepsilon \mathrm{e}^{-\ell \lambda_{0} / \varepsilon}, \varepsilon^{1-\alpha} \mathrm{e}^{-\ell \lambda_{0} / \varepsilon}\right]$. So, in what follows we set

DEFINITION 12. - Let us define

$$
\begin{aligned}
& \delta_{0}:=\sup _{\left.\left.z \in \mathfrak{B}_{\ell}, \varepsilon \in\right] 0,1\right]}\left(\left|u_{0}^{h}(z)\right|+\varepsilon\left|Y_{0}^{h}(z)\right|_{\widehat{\mathbb{\pi}_{\varepsilon} \mathbb{D}}}\right) \text { and } \varepsilon_{0}:=\varepsilon_{0}\left(\delta_{0}\right), \\
& \varphi_{0}:=\sup _{\left.\varepsilon \in] 0, \varepsilon_{0}\right], A_{0} \in\left[0, \delta_{1}[\right.}\left(\frac{4}{\rho\left[\lambda_{\varepsilon}+\varepsilon \gamma_{1}\left(0,0, A_{0}^{2}, \varepsilon\right)\right]}\right),
\end{aligned}
$$

where $\varepsilon_{0}\left(\delta_{0}\right)$ is given by the Nonlocal Normal Form Lemma 3 and let us set

$$
\left.A_{0}=A_{0}^{\star} \mathrm{e}^{-\frac{\lambda_{0} \ell}{\varepsilon}} \quad \text { with } A_{0}^{\star} \in\right]-\frac{1}{2} \delta_{1}, \frac{1}{2} \delta_{1}[.
$$

We also define 
DEFINITION 13. - Let $\mathfrak{H}_{\ell}^{\alpha}=H_{\ell, 1}^{\alpha} \times H_{\ell, 2}^{\alpha} \times H_{\ell, 2}^{1, \alpha} \times H_{\underline{\underline{x}}_{\varepsilon} \mathbb{D}, w}^{\alpha}$ equipped with the following norm

$$
\|\mathfrak{h}\|_{\mathfrak{H}_{\ell}^{\alpha}}=\left\|\left(q_{1}, q_{2}\right)\right\|_{\mathfrak{Q}_{\ell}^{\alpha}}+\|u\|_{H_{\ell, 2}^{1, \alpha}}+\|Y\|_{H_{\mathbb{\pi}_{\varepsilon} \mathbb{D}, w}^{\alpha}},
$$

where $\mathfrak{h}=\left(q_{1}, q_{2}, u, Y\right)$ and

$$
\begin{aligned}
\left\|\left(q_{1}, q_{2}\right)\right\|_{\mathfrak{Q}_{\ell}^{\alpha}}= & \mathrm{e}^{-\frac{\lambda_{0} \ell}{\varepsilon}}\left(\left\|q_{1}\right\|_{H_{\ell, 1}^{\alpha}}+\left\|q_{2}\right\|_{H_{\ell, 2}^{\alpha}}\right)+\left\|\left(i q_{1}+q_{2}\right) \mathrm{e}^{i \frac{\lambda_{0} z}{\varepsilon}}\right\|_{H_{\ell, 1}^{\alpha}} \\
& +\left\|\left(-i q_{1}+q_{2}\right) \mathrm{e}^{-i \frac{\lambda_{0} z}{\varepsilon}}\right\|_{H_{\ell, 1}^{\alpha}}
\end{aligned}
$$

Then, we define

$$
\mathfrak{B} \mathfrak{H}_{\ell}^{\alpha}\left(d_{0}, d_{1}\right)=\left\{\mathfrak{h} \in \mathfrak{H}_{\ell}^{\alpha} /\|u\|_{H_{\ell, 2}^{1, \alpha}}+\|Y\|_{H_{\underline{\pi}_{\varepsilon}}^{\alpha} \mathbb{D}, w}<d_{0},\left\|\left(q_{1}, q_{2}\right)\right\|_{\left.\mathfrak{Q}_{\ell}^{\alpha}<d_{1}\right\} .}\right.
$$

Observe that there is no $\varepsilon$ in front of $\|Y\|_{H_{\mathbb{\pi}_{\varepsilon} \mathbb{D}, w}^{\alpha}}$ in the definition of $\mathfrak{B H}_{\ell}^{\alpha}\left(d_{0}, d_{1}\right)$.

With this choice of parameters we check

LEMMA 14. -

(a) There exists $c$ such that for every $\left.\left.z \in \mathfrak{B}_{\ell}, \varphi \in\left[-\varphi_{0}, \varphi_{0}\right], \varepsilon \in\right] 0, \varepsilon_{0}\right]$ and every $\left.A_{0}^{\star} \in\right]-\frac{1}{2} \delta_{1}, \frac{1}{2} \delta_{1}\left[,\left|\mathcal{I} m\left(\psi_{\varphi}(z)\right)\right| \leqslant \frac{\lambda_{0} \ell}{\varepsilon}+c\right.$ holds.

(b) There exists $\left.\delta_{2} \in\right] 0, \frac{1}{2} \delta_{1}\left[\right.$ such that for every $\left.\left.z \in \mathfrak{B}_{\ell}, \varphi \in\left[-\varphi_{0}, \varphi_{0}\right], \varepsilon \in\right] 0, \varepsilon_{0}\right]$ and every $A_{0}^{\star} \in\left[-\delta_{2}, \delta_{2}\right],\left|A_{0} \mathrm{e}^{i \psi_{\varphi}(z)}\right|<\frac{1}{2} \delta_{1}$.

(c) There exists $\left.\delta_{3} \in\right] 0, \delta_{1}\left[\right.$ such that for every $\left.\left.z \in \mathfrak{B}_{\ell}, \varphi \in\left[-\varphi_{0}, \varphi_{0}\right], \varepsilon \in\right] 0, \varepsilon_{0}\right]$ and every $\mathfrak{h} \in \mathfrak{B H}_{\ell}^{\alpha}\left(\delta_{0}, \delta_{3}\right)$

$$
\left|\left(q_{2}+i q_{1}\right) \mathrm{e}^{i \psi_{\varphi}(z)}\right| \leqslant \frac{1}{2} \delta_{1}, \quad\left|\left(q_{2}-i q_{1}\right) \mathrm{e}^{-i \psi_{\varphi}(z)}\right| \leqslant \frac{1}{2} \delta_{1} .
$$

(d) For every $\left.\left.z \in \mathfrak{B}_{\ell}, \varphi \in\left[-\varphi_{0}, \varphi_{0}\right], \varepsilon \in\right] 0, \varepsilon_{0}\right], A_{0}^{\star} \in\left[-\delta_{2}, \delta_{2}\right]$ and every $\mathfrak{h} \in$ $\mathfrak{B H}_{\ell}^{\alpha}\left(\delta_{0}, \delta_{3}\right)$, the corresponding $\mathrm{H}_{A_{0}, \varphi, \varepsilon}$ given by (17) and (18) lies in $\mathrm{BE}_{\ell}^{\alpha}\left(\delta_{0}, \delta_{1}\right)$.

So, we are looking for $\varphi \in\left[-\varphi_{0}, \varphi_{0}\right]$ and for an homoclinic connection to $0 \mathfrak{h}=$ $\left(q_{1}, q_{2}, u, Y\right)$ in $\mathfrak{B H}_{\ell}^{\alpha}\left(\delta_{0}, \delta_{3}\right)$ which is reversible, i.e.

$$
\mathfrak{S h}(z)=\mathfrak{h}(-z) \quad \text { where } \mathfrak{S}\left(q_{1}, q_{2}, u, Y\right)=\left(-q_{1}, q_{2}, u, S Y\right) .
$$

The new system satisfied by $\mathfrak{h}=\left(q_{1}, q_{2}, u, Y\right)$ and $\varphi$ reads

$$
\begin{aligned}
& \frac{d q_{1}}{d z}=\left(A_{0}+q_{2}\right)\left[\gamma_{1}(u, 0,0, \varepsilon)-\varphi \rho_{0}\right]+R_{q_{1}}^{\prime}, \\
& \frac{d q_{2}}{d z}=-q_{1}\left[\gamma_{1}(u, 0,0, \varepsilon)-\varphi \rho_{0}\right]+R_{q_{2}}^{\prime}, \\
& \rho \mathcal{H}\left[\frac{d u}{d z}\right]+u+\frac{3}{2} u^{2}=\mathcal{R}_{\varepsilon, u}^{\prime}+R_{\varepsilon, u}^{\prime}+c_{0}, \\
& Y-\mathcal{T}_{0}[u]=\mathcal{R}_{\varepsilon, Y}^{\prime},
\end{aligned}
$$

where 


$$
\begin{aligned}
& \rho_{0}(z)=\left(\lambda_{\varepsilon}+\varepsilon \gamma_{1}\left(0,0, A_{0}^{2}, \varepsilon\right)\right) \frac{\rho^{2}}{z^{2}+\rho^{2}}, \\
& R_{q_{1}}^{\prime}=\frac{1}{2 i}\left(R_{\varepsilon, A}^{\prime} \mathrm{e}^{-i \psi_{\varphi}}-R_{\varepsilon, \tilde{A}}^{\prime} \mathrm{e}^{i \psi_{\varphi}}\right)+\left(A_{0}+q_{2}\right) \Delta_{\gamma}^{\prime}, \\
& R_{q_{2}}^{\prime}=\frac{1}{2}\left(R_{\varepsilon, A}^{\prime} \mathrm{e}^{-i \psi_{\varphi}}+R_{\varepsilon, \tilde{A}}^{\prime} \mathrm{e}^{i \psi_{\varphi}}\right)-q_{1} \Delta_{\gamma}^{\prime},
\end{aligned}
$$

with

$$
\Delta_{\gamma}^{\prime}=\left\{\gamma_{1}\left(u, \varepsilon p_{1}^{*}(Y), A \widetilde{A}, \varepsilon\right)-\gamma_{1}(u, 0,0, \varepsilon)-\gamma_{1}\left(0,0, A_{0}^{2}, \varepsilon\right)\right\}
$$

where we put a prime when we need to replace $A$ by $\left(A_{0}+q_{2}+i q_{1}\right) \mathrm{e}^{i \psi_{\varphi}}, \widetilde{A}$ by $\left(A_{0}+q_{2}-i q_{1}\right) \mathrm{e}^{-i \psi_{\varphi}}$, and where we choose the constant $c_{0}$ such that $\left(q_{1}, q_{2}, Y, u\right)=0$ cancels $\mathcal{R}_{\varepsilon, u}^{\prime}+R_{\varepsilon, u}^{\prime}+c_{0}$, i.e.

$$
c_{0}=-R_{\varepsilon, u}\left(A_{0}^{2}\right)
$$

\subsection{Strategy of proof}

We look for a reversible homoclinic connection $\mathfrak{h}=\left(q_{1}, q_{2}, u, Y\right)$ to 0 of the full Eq. (19) under the form

$$
\mathfrak{h}=\mathfrak{h}_{0, \varepsilon}+\mathfrak{h}_{1}
$$

with

$$
\mathfrak{h}_{0, \varepsilon}=\left(0,0, u_{0}^{h}, Y_{0}^{h}\right) \in \mathfrak{B H}_{\ell}^{\alpha}\left(\frac{1}{2} \delta_{0}, 0\right), \quad \mathfrak{h}_{1}=\left(q_{1}, q_{2}, w, Z\right)
$$

More precisely, we look for $\varphi \in\left[-\varphi_{0}, \varphi_{0}\right]$ and $\mathfrak{h}_{1} \in \mathfrak{B H}_{\ell}^{\alpha}\left(\frac{1}{2} \delta_{0}, \delta_{3}\right)$, which satisfies

$$
\mathbf{L}_{\varphi}(z) \mathfrak{h}_{1}=\mathbf{G}\left(\mathfrak{h}_{1}, A_{0}^{\star}, \varphi, \varepsilon\right),
$$

where

$$
\mathbf{L}_{\varphi}(z) \mathfrak{h}_{1}=\mid \begin{aligned}
& \frac{d q_{1}}{d z}-\left[\gamma_{1}\left(u_{0}^{h}, 0,0, \varepsilon\right)-\varphi \rho_{0}\right] q_{2} \\
& \frac{d q_{2}}{d z}+\left[\gamma_{1}\left(u_{0}^{h}, 0,0, \varepsilon\right)-\varphi \rho_{0}\right] q_{1} \\
& \rho \mathcal{H}\left[\frac{d w}{d z}\right]+w+3 u_{0}^{h} w \\
& Z-\mathcal{T}_{0} w
\end{aligned}
$$

and

$$
\mathbf{G}=\left(G_{q_{1}}, G_{q_{2}}, \mathcal{G}_{w}, \mathcal{G}_{Z}\right)
$$

with

$$
\begin{aligned}
G_{q_{1}}= & A_{0}\left(\gamma_{1}\left(u_{0}^{h}+w, 0,0, \varepsilon\right)-\varphi \rho_{0}\right) \\
& +q_{2}\left(\gamma_{1}\left(u_{0}^{h}+w, 0,0, \varepsilon\right)-\gamma_{1}\left(u_{0}^{h}, 0,0, \varepsilon\right)\right)+R_{q_{1}}^{\prime},
\end{aligned}
$$




$$
\begin{aligned}
G_{q_{2}} & =-q_{1}\left(\gamma_{1}\left(u_{0}^{h}+w, 0,0, \varepsilon\right)-\gamma_{1}\left(u_{0}^{h}, 0,0, \varepsilon\right)\right)+R_{q_{2}}^{\prime}, \\
\mathcal{G}_{w} & =\mathcal{R}_{\varepsilon, u}^{\prime}+R_{\varepsilon, u}^{\prime}-R_{\varepsilon, u}^{\prime}\left(A_{0}^{2}\right)-\frac{3}{2} w^{2}, \\
\mathcal{G}_{Z} & =\mathcal{R}_{\varepsilon, Y}^{\prime} .
\end{aligned}
$$

Here the reversibility comes from the invariance of the system under the symmetry

$$
\left(z, q_{1}, q_{2}, w, Z\right) \mapsto\left(-z,-q_{1}, q_{2}, w, S Z\right) .
$$

Moreover the map

$$
\left(\varphi, q_{1}, q_{2}, w, Z\right) \mapsto\left(G_{q_{1}}, G_{q_{2}}, \mathcal{G}_{w}, \mathcal{G}_{Z}\right)
$$

is analytic from $]-\varphi_{0}, \varphi_{0}\left[\times \mathfrak{B H}_{\ell}^{\alpha}\left(\frac{1}{2} \delta_{0}, \delta_{3}\right)\right.$ to $H_{\ell, 2}^{\alpha} \times H_{\ell, 3}^{\alpha} \times H_{\ell, 2}^{\alpha} \times H_{\underline{\pi}_{\varepsilon}}^{\alpha} \mathbb{D}, w$. For finding homoclinic connection to 0 of (20) we proceed in several steps:

Step 1. In Section 4.3 we consider the affine equation

$$
\mathbf{L}_{\varphi}(z) \mathfrak{h}=\mathbf{F} .
$$

More precisely we prove that for any antireversible $\mathbf{F} \in H_{\ell, 2}^{\alpha} \times H_{\ell, 3}^{\alpha} \times H_{\ell, 2}^{\alpha} \times H_{\underline{\pi}_{\varepsilon} \mathbb{D}, w}^{\alpha}$ there exists a reversible solution $\mathfrak{h}$ in $\mathfrak{H}_{\ell}^{\alpha}$ if and only if $\mathbf{F}$ satisfies the solvability condition

$$
\int_{0}^{\infty}\left\langle r_{-}(\underline{x}), \mathbf{F}(\underline{x})\right\rangle d \underline{x}=0,
$$

where $r_{-}$is given by

$$
r_{-}=(\cos \Gamma,-\sin \Gamma, 0,0),
$$

with

$$
\Gamma(z)=\int_{[0, z]}\left(\gamma_{1}\left(u_{0}^{h}(\tau), 0,0, \varepsilon\right)-\varphi \rho_{0}(\tau)\right) d \tau .
$$

So, a necessary condition for the existence of a solution $\mathfrak{h}_{1}$ of (20) in $\mathfrak{B} \mathfrak{H}_{\ell}^{\alpha}\left(\frac{1}{2} \delta_{0}, \delta_{3}\right)$ is that

$$
J\left(\mathfrak{h}_{1}, \varphi, A_{0}^{\star}, \varepsilon\right)=0,
$$

where

$$
J=\int_{0}^{\infty}\left\langle r_{-}, \mathbf{G}\left(\mathfrak{h}_{1}, A_{0}^{\star}, \varphi, \varepsilon\right)\right\rangle d \underline{x} .
$$

Step 2. For studying $J$ and (20), we need precise estimates of $G_{q_{1}}, G_{q_{2}}, \mathcal{G}_{w}, \mathcal{G}_{Z}$. They are given in Section 4.4.

Step 3. In Section 4.5, we study the solvability function $J$ and we compute its principal part. $J$ happens to be an oscillatory integral and its study is based on the Exponential Lemma 4 . This is why, we had to complexify the problem. 
Step 4. In Section 4.6.1, we introduce the modified equation

$$
\mathbf{L}_{\varphi}(z) \mathfrak{h}_{1}=\mathbf{G}^{\perp}\left(\mathfrak{h}_{1}, A_{0}^{\star}, \varphi, \varepsilon\right),
$$

where

$$
\mathbf{G}^{\perp}=\mathbf{G}-\frac{2}{\sqrt{\pi}} J \mathrm{e}^{-z^{2}} r_{-}(z) .
$$

The term $\mathbf{G}^{\perp}$ has been designed so that, for every $\varepsilon, A_{0}^{\star}, \varphi, \mathfrak{h}_{1}$

$$
\int_{0}^{\infty}\left\langle r_{-}, \mathbf{G}^{\perp}\left(\mathfrak{h}_{1}, A_{0}^{\star}, \varphi, \varepsilon\right)\right\rangle d \underline{x}=0 .
$$

Then, using the implicit function theorem, we prove that for any $\varphi$ and any sufficiently small $\left|A_{0}^{\star}\right|, \varepsilon$ the system (20) admits a solution $\mathfrak{h}_{1, \varepsilon, A_{0}^{\star}, \varphi}$ in $\mathfrak{B H}_{\ell}^{\alpha}\left(\frac{1}{2} \delta_{0}, \delta_{3}\right)$ satisfying

$$
\left\|\mathfrak{h}_{1, \varphi, A_{0}^{\star}, \varepsilon}\right\|_{\mathfrak{H}_{\ell}^{\alpha}} \leqslant c\left(\varepsilon^{1-\alpha}+\left|A_{0}^{\star}\right|\right) .
$$

Step 5. Finally, in Section 4.6.2, using the study of $J$ made in 4.5, we prove that for $0<\alpha \leqslant \frac{1}{2}$, there exist $c_{\star}, \varepsilon_{\star}$ such that for every $0<\varepsilon<\varepsilon_{\star}, A_{0}^{\star} \in\left[c_{\star} \varepsilon, \varepsilon^{1-\alpha}\right]$ there exists $\varphi\left(\varepsilon, A_{0}^{\star}\right)$ such that

$$
J\left[\mathfrak{h}_{1, A_{0}^{\star}, \varphi\left(\varepsilon, A_{0}^{\star}\right), \varepsilon}, \varphi\left(\varepsilon, A_{0}^{\star}\right), A_{0}^{\star}, \varepsilon\right]=0 .
$$

Hence, $\mathfrak{h}_{1, A_{0}^{\star}, \varphi\left(\varepsilon, A_{0}^{\star}\right), \varepsilon}$ is a reversible solution of $(20)$ in $\mathfrak{B H}_{\ell}^{\alpha}\left(\frac{1}{2} \delta_{0}, \delta_{3}\right)$ which gives the existence of an homoclinic connection to 0 for (19) under the form

$$
\mathfrak{h}=\mathfrak{h}_{0, \varepsilon}+\mathfrak{h}_{1, A_{0}^{\star}, \varphi\left(\varepsilon, A_{0}^{\star}\right), \varepsilon} .
$$

At this last step we have only considered positive values of $A_{0}^{\star}$ since the solution obtained for $A_{0}^{\star}<0$ are the same as the one found for $A_{0}^{\star}>0$ thanks to the undetermination on the form of the parametrization of the bifurcating solution given by (16).

\subsection{Linearized system around the homoclinic connection of the truncated system}

This subsection is devoted to the study of the affine equation

$$
\mathbf{L}_{\varphi}(z) \mathfrak{h}=\mathbf{F},
$$

for any given $\mathbf{F}=\left(F_{q_{1}}, F_{q_{2}}, F_{Z}, F_{w}\right) \in H_{\ell, 2}^{\alpha} \times H_{\ell, 3}^{\alpha} \times H_{\ell, 2}^{\alpha} \times H_{\underline{\pi}_{s} \mathbb{D}, w}^{\alpha}$ which is antireversible, i.e. such that $F_{q_{1}}$ and $F_{w}$ are even, $F_{q_{2}}$ is odd, while $F_{Z}$ is reversible (i.e. $\left.S F_{Z}(-z)=F_{Z}(z)\right)$. Eq. (20) reads

$$
\begin{aligned}
& \frac{d q_{1}}{d z}-\left(\gamma_{1}\left(u_{0}^{h}, 0,0, \varepsilon\right)-\varphi \rho_{0}\right) q_{2}=F_{q_{1}}, \\
& \frac{d q_{2}}{d z}+\left(\gamma_{1}\left(u_{0}^{h}, 0,0, \varepsilon\right)-\varphi \rho_{0}\right) q_{1}=F_{q_{2}},
\end{aligned}
$$




$$
\begin{aligned}
& \rho \mathcal{H}\left[\frac{d w}{d z}\right]+w+3 u_{0}^{h} w=F_{w}, \\
& Z-\mathcal{T}_{0}[w]=F_{Z} .
\end{aligned}
$$

Let us first show the inversion for the two first coordinates. Let us consider a basis of solutions of the homogeneous system in $\left(q_{1}, q_{2}\right)$

$$
\begin{aligned}
& r_{+}(z)=(\sin \Gamma(z), \cos \Gamma(z), 0,0), \\
& r_{-}(z)=(\cos \Gamma(z),-\sin \Gamma(z), 0,0), \\
& \Gamma(z)=\int_{[0, z]}\left(\gamma_{1}\left(u_{0}^{h}(\tau), 0,0, \varepsilon\right)-\varphi \rho_{0}(\tau)\right) d \tau
\end{aligned}
$$

then $r_{+}$is reversible, while $r_{-}$is antireversible, and $\Gamma$ is odd and may be also written as

$$
\Gamma(z)=\int_{[0, z]} \gamma_{1}\left(u_{0}^{h}(\tau), 0,0, \varepsilon\right) d \tau-\varphi \rho\left[\lambda_{\varepsilon}+\varepsilon \gamma_{1}\left(0,0, A_{0}^{2}, \varepsilon\right)\right] \arctan (z / \rho) .
$$

Let us denote $\langle\cdot, \cdot\rangle$ the canonical scalar product in $\mathbb{C}^{2}$ : for $q=\left(q_{1}, q_{2}\right)$ and $q^{\prime}=\left(q_{1}^{\prime}, q_{2}^{\prime}\right)$, $\left\langle q, q^{\prime}\right\rangle=\overline{q_{1}} q_{1}^{\prime}+\overline{q_{2}} q_{2}^{\prime}$. We identify $\left(\mathbb{C}^{2}\right)^{*}$ with $\mathbb{C}^{2}$ by $q \mapsto\langle q, \cdot\rangle_{*}:=\langle\bar{q}, \cdot\rangle$. We then show

LEMMA 15. - Let us consider the affine system

$$
\begin{aligned}
& \frac{d q_{1}}{d z}=q_{2}\left(\gamma_{1}\left(u_{0}^{h}, 0,0, \varepsilon\right)-\varphi \rho_{0}\right)+F_{q_{1}}, \\
& \frac{d q_{2}}{d z}=-q_{1}\left(\gamma_{1}\left(u_{0}^{h}, 0,0, \varepsilon\right)-\varphi \rho_{0}\right)+F_{q_{2}},
\end{aligned}
$$

with $F_{q}=\left(F_{q_{1}}, F_{q_{2}}\right) \in H_{\ell, 2}^{\alpha} \times H_{\ell, 3}^{\alpha}$, antireversible $\left(F_{q_{1}}\right.$ even, $F_{q_{2}}$ odd $)$. This system has a unique reversible, holomorphic solution $\left(q_{1}, q_{2}\right)=\mathcal{F}_{q}\left(F_{q}\right),\left(q_{1}\right.$ odd, $q_{2}$ even $)$ tending towards 0 at infinity, if and only if (we identify $r_{-}$with its two first components)

$$
\int_{0}^{\infty}\left\langle r_{-}(\underline{x}), F_{q}(\underline{x})\right\rangle_{*} d \underline{x}=0
$$

We have

$$
\mathcal{F}_{q}\left(F_{q}\right)(z)=-r_{+}(z) \int_{z+\mathbb{R}^{+}}\left\langle r_{+}(\tau), F_{q}(\tau)\right\rangle_{*} d \tau-r_{-}(z) \int_{z+\mathbb{R}^{+}}\left\langle r_{-}(\tau), F_{q}(\tau)\right\rangle_{*} d \tau .
$$

Moreover, there exists $c$ such that for every $\left.\left.\varphi \in\left[-\varphi_{0}, \varphi_{0}\right], \varepsilon \in\right] 0, \varepsilon_{0}\right]$ and every $F_{q}=$ $\left(F_{q_{1}}, F_{q_{2}}\right) \in H_{\ell, 2}^{\alpha} \times H_{\ell, 3}^{\alpha}$ satisfying $(29), \mathcal{F}_{q}\left(F_{q}\right)$ lies in $H_{\ell, 1}^{\alpha} \times H_{\ell, 2}^{\alpha}$ and satisfy

(ii) $\quad\left\|\left(i \mathcal{F}_{q_{1}}\left(F_{q}\right)+\mathcal{F}_{q_{2}}\left(F_{q}\right)\right) \mathrm{e}^{i \frac{\lambda_{0} z}{\varepsilon}}\right\|_{H_{\ell, 1}^{\alpha}} \leqslant c\left(\left\|F_{A}\right\|_{H_{\ell, 2}^{\alpha}}+\left\|F_{\tilde{A}}\right\|_{H_{\ell, 2}^{\alpha}}\right)$,

(iii) $\left\|\left(-i \mathcal{F}_{q_{1}}\left(F_{q}\right)+\mathcal{F}_{q_{2}}\left(F_{q}\right)\right) \mathrm{e}^{-i \frac{\lambda_{0} z}{\varepsilon}}\right\|_{H_{\ell, 1}^{\alpha}} \leqslant c\left(\left\|F_{A}\right\|_{H_{\ell, 2}^{\alpha}}+\left\|F_{\tilde{A}}\right\|_{H_{\ell, 2}^{\alpha}}\right)$,

where $F_{A}=\left(i F_{q_{1}}+F_{q_{2}}\right) \mathrm{e}^{i \frac{\lambda_{0} z}{\varepsilon}}$ and $F_{\tilde{A}}=\left(-i F_{q_{1}}+F_{q_{2}}\right) \mathrm{e}^{-i \frac{\lambda_{0} z}{\varepsilon}}$. 


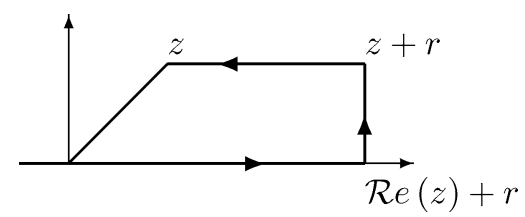

Fig. 7. Path $\Gamma_{r}$.

Proof. - Step 1. Explicit formula. Variation of constants method leads to

$$
\begin{aligned}
\mathcal{F}_{q}\left(F_{q}\right)(z)= & \left(c_{+}+\int_{[0, z]}\left\langle r_{+}(\tau), F_{q}(\tau)\right\rangle_{*} d \tau\right) r_{+}(z) \\
& +\left(c_{-}+\int_{[0, z]}\left\langle r_{-}(\tau), F_{q}(\tau)\right\rangle_{*} d \tau\right) r_{-}(z)
\end{aligned}
$$

We check that $\mathcal{F}_{q}\left(F_{q}\right)$ is reversible if and only if $c_{-}=0$ and that if $\mathcal{F}_{q}\left(F_{q}\right)(\underline{x}) \underset{x \rightarrow+\infty}{\longrightarrow} 0$, then necessarily

$$
\begin{aligned}
& c_{+}=-\int_{0}^{\infty}\left\langle r_{+}(\tau), F_{q}(\tau)\right\rangle_{*} d \tau, \\
& c_{-}=-\int_{0}^{\infty}\left\langle r_{-}(\tau), F_{q}(\tau)\right\rangle_{*} d \tau .
\end{aligned}
$$

Hence, we deduce the compatibility condition (29), and the explicit form of $\mathcal{F}_{q}\left(F_{q}\right)$ using the holomorphy of the solutions and the integral path $\Gamma_{r}$ drawn on Fig. 7.

So, we assume now that $F_{q}$ is an antireversible function lying in $H_{\ell, 2}^{\alpha} \times H_{\ell, 3}^{\alpha}$ and satisfying the compatibility condition (29).

Step 2. Estimates in $H_{\ell, 1}^{\alpha}$. The two explicit formulas (30), (31) ensure that $\mathcal{F}_{q}\left(F_{q}\right)$ is holomorphic in $\mathfrak{B}_{\ell}$. Moreover, using (28), and the reversibility of $\mathcal{F}_{q}\left(F_{q}\right)$ we get that there exists $c$ such that for every $\varphi \in\left[-\varphi_{0}, \varphi_{0}\right]$ and every $\left.\left.\varepsilon \in\right] 0, \varepsilon_{0}\right]$

$$
\left\|\mathcal{F}_{q_{1}}\left(F_{q}\right)\right\|_{H_{\ell, 1}^{\alpha}}+\left\|\mathcal{F}_{q_{2}}\left(F_{q}\right)\right\|_{H_{\ell, 1}^{\alpha}} \leqslant c\left(\left\|F_{q_{1}}\right\|_{H_{\ell, 2}^{\alpha}}+\left\|F_{q_{2}}\right\|_{H_{\ell, 3}^{\alpha}}\right) \text {. }
$$

Step 3. Estimates of $\mathcal{F}_{q_{2}}\left(F_{q}\right)$ in $H_{\ell, 2}^{\alpha}$. Since $\mathcal{F}_{q_{2}}\left(F_{q}\right)(z) \underset{\mathcal{R} e(z) \rightarrow+\infty}{\longrightarrow} 0$, we have

$$
\mathcal{F}_{q_{2}}\left(F_{q}\right)(z)=-\int_{0}^{+\infty} \frac{d}{d z}\left(\mathcal{F}_{q_{2}}\left(F_{q}\right)\right)(z+\tau) d \tau
$$

Moreover, $\mathcal{F}_{q_{2}}\left(F_{q}\right)$ is even, lies in $H_{\ell, 1}^{\alpha}$ and satisfies (28). Hence, $\frac{d \mathcal{F}_{q_{2}}\left(F_{q}\right)}{d z}$ lies in $H_{\ell, 3}^{\alpha}$; $\mathcal{F}_{q_{2}}\left(F_{q}\right)$ lies in $H_{\ell, 2}^{\alpha}$ and there exists $c$ such that for every $\left.\left.\varphi \in\left[-\varphi_{0}, \varphi_{0}\right], \varepsilon \in\right] 0, \varepsilon_{0}\right]$,

$$
\left\|\mathcal{F}_{q_{2}}\left(F_{q}\right)\right\|_{H_{\ell, 2}^{\alpha}} \leqslant c\left(\left\|F_{q_{1}}\right\|_{H_{\ell, 2}^{\alpha}}+\left\|F_{q_{2}}\right\|_{H_{\ell, 3}^{\alpha}}\right)
$$


Step 4. Estimates of $\mathcal{F}_{A}\left(F_{q}\right)=\left(i \mathcal{F}_{q_{1}}\left(F_{q}\right)+\mathcal{F}_{q_{2}}\left(F_{q}\right)\right) \mathrm{e}^{i \lambda_{0} z / \varepsilon}$ and $\mathcal{F}_{\tilde{A}}\left(F_{q}\right)=$ $\left(-i \mathcal{F}_{q_{1}}\left(F_{q}\right)+\mathcal{F}_{q_{2}}\left(F_{q}\right)\right) \mathrm{e}^{-i \lambda_{0} z / \varepsilon}$. We check that $\mathcal{F}_{A}\left(F_{q}\right)$ satisfies

$$
\frac{d \mathcal{F}_{A}\left(F_{q}\right)}{d z}=i \mathcal{F}_{A}\left(F_{q}\right)\left[\frac{\lambda_{0}}{\varepsilon}+\gamma_{1}\left(u_{0}^{h}, 0,0, \varepsilon\right)-\varphi \rho_{0}\right]+F_{A}
$$

Since $\mathcal{F}_{A}\left(F_{q}\right)(z) \underset{\mathcal{R} e(z) \rightarrow+\infty}{\longrightarrow} 0$, we get

$$
\mathcal{F}_{A}\left(F_{q}\right)(z)=-\int_{0}^{+\infty} \mathrm{e}^{i \frac{\lambda_{0}}{\varepsilon} \tau+i \Gamma(z+\tau)-i \Gamma(z)} F_{A}(z+\tau) d \tau
$$

and

$$
\mathcal{F}_{A}\left(F_{q}\right)(z)=\int_{-\infty}^{0} \mathrm{e}^{-\left(i \frac{\lambda_{0}}{\varepsilon} \tau+i \Gamma(-z+\tau)-i \Gamma(-z)\right)} F_{\tilde{A}}(-z+\tau) d \tau
$$

because of reversibility. Hence, there exists $c$ such that for every $\varphi \in\left[-\varphi_{0}, \varphi_{0}\right], \varepsilon \in$ ] $\left.0, \varepsilon_{0}\right]$,

$$
\left\|\mathcal{F}_{A}\left(F_{q}\right)\right\|_{H_{\ell, 1}^{\alpha}} \leqslant c\left(\left\|F_{A}\right\|_{H_{\ell, 2}^{\alpha}}+\left\|F_{\tilde{A}}\right\|_{H_{\ell, 2}^{\alpha}}\right) .
$$

Similarly we prove that there exists $c$ such that for every $\left.\left.\varphi \in\left[-\varphi_{0}, \varphi_{0}\right], \varepsilon \in\right] 0, \varepsilon_{0}\right]$,

$$
\left\|\mathcal{F}_{\tilde{A}}\left(F_{q}\right)\right\|_{H_{\ell, 1}^{\alpha}} \leqslant c\left(\left\|F_{A}\right\|_{H_{\ell, 2}^{\alpha}}+\left\|F_{\tilde{A}}\right\|_{H_{\ell, 2}^{\alpha}}\right) .
$$

It remains to invert the second part of system (20) with respect to $(w, Z)$. This is given by the following

LEMMA 16. - Let consider the affine system in $H_{\ell, 2}^{\alpha} \times H_{\underline{\pi}_{\varepsilon}}^{\alpha} \mathbb{D}, w$

$$
\begin{aligned}
& \rho \mathcal{H}\left[\frac{d w}{d z}\right]+w+3 u_{0}^{h} w=F_{w}, \\
& Z-\mathcal{T}_{0}[w]=F_{Z},
\end{aligned}
$$

where $F_{Z}$ is reversible, and $F_{w}$ is even. Then, there is a unique reversible solution $(w, Z)$ such that $\left(F_{w}, F_{Z}\right) \mapsto(w, Z)$ is a bounded linear map:

$$
H_{\ell, 2}^{\alpha} \times H_{\underline{\pi}_{\varepsilon} \mathbb{D}, w}^{\alpha} \rightarrow H_{\ell, 2}^{1, \alpha} \times H_{\underline{\pi}_{\varepsilon}}^{\alpha} \mathbb{D}, w
$$

with an estimate

$$
\|w\|_{H_{\ell, 2}^{1, \alpha}}+\|Z\|_{H_{\mathbb{\pi}_{\varepsilon} \mathbb{\mathbb { D }}, w}^{\alpha}} \leqslant c\left(\left\|F_{w}\right\|_{H_{\ell, 2}^{\alpha}}+\left\|F_{Z}\right\|_{H_{\mathbb{\pi}_{\varepsilon} \mathbb{\mathbb { D }}, w}^{\alpha}}\right) .
$$

Proof. - Since $\mathcal{T}_{0}$ is a bounded linear operator from $H_{\ell, 2}^{1, \alpha}$ to $H_{\underline{\pi}_{\varepsilon} \mathbb{D}, w}^{\alpha}$, it is sufficient to solve the equation for $w$, which is the linearized Benjamin-Ono equation. It is shown 
in [1] that if $F_{w} \in B_{2}^{\alpha}(\mathbb{R})$, then the solution $w$ of the linearized B-O equation lies in $B_{2}^{1, \alpha}(\mathbb{R})$, with

$$
\|w\|_{B_{2}^{1, \alpha}(\mathbb{R})} \leqslant c\left\|F_{w}\right\|_{B_{2}^{\alpha}(\mathbb{R})} .
$$

Let us show that this estimate holds when we replace $\left(B_{2}^{1, \alpha}(\mathbb{R}), B_{2}^{\alpha}(\mathbb{R})\right)$ by $\left(H_{\ell, 2}^{1, \alpha}, H_{\ell, 2}^{\alpha}\right)$.

Step 1 . Let us define the linear operator $\mathcal{A}$ defined on $H_{\ell, 2}^{1, \alpha}$ by

$$
\mathcal{A}[w]=\rho \mathcal{H}\left[\frac{d w}{d z}\right]+w .
$$

To show that it is an isomorphism of Banach spaces from $H_{\ell, 2}^{1, \alpha}$ onto $H_{\ell, 2}^{\alpha}$ we first check that is is a bounded linear operator from $H_{\ell, 2}^{1, \alpha}$ to $H_{\ell, 2}^{\alpha}$. For that purpose we introduce $\phi \in C^{\infty}(\mathbb{R})$ such that $\phi(s)=1$ for $|s| \leqslant 1$, and $\phi(s)=0$ for $|s| \geqslant 2$, then we have

$$
\begin{aligned}
\mathcal{A}[w](z)=\rho \mathcal{H}\left[\frac{d w}{d z}\right](z)+w(z)= & w(z)+\text { p.v. } \frac{\rho}{\pi} \int_{\mathbb{R}} \phi(s) K(s) \frac{d w}{d z}(z-s) d s \\
& +\frac{\rho}{\pi} \int_{\mathbb{R}}([1-\phi(s)] K(s))_{s}^{\prime} w(z-s) d s,
\end{aligned}
$$

where $K(s)=1 / s$. We now observe that both $\phi K$ and $([1-\phi(s)] K(s))_{s}^{\prime}$ satisfy the hypothesis on the kernel $K$ in Lemma 7, hence $\mathcal{A}$ is a bounded linear operator from $H_{\ell, 2}^{1, \alpha}$ to $H_{\ell, 2}^{\alpha}$.

Moreover we can compute explicitly the inverse of $\mathcal{A}$ which is given by

$$
\mathcal{A}^{-1}[f](z)=\int_{\mathbb{R}} K_{1}(s) f(z-s) d s
$$

where

$$
K_{1}(s)=\frac{1}{\rho \pi} \int_{0}^{+\infty} \frac{\tau \mathrm{e}^{-\tau / \rho}}{s^{2}+\tau^{2}} d \tau
$$

Then observing that

$$
\frac{d\left(\mathcal{A}^{-1}[w]\right)}{d z}(z)=\int_{\mathbb{R}} K_{1}^{\prime}(s) f(z-s) d s
$$

and that $K_{1}$ and $K_{1}^{\prime}$ satisfy the assumptions of Lemma 7 with $\mathbb{E}=\mathbb{R}$, we finally obtain that $\mathcal{A}$ is an isomorphism of Banach space from $H_{\ell, 2}^{1, \alpha}$ onto $H_{\ell, 2}^{\alpha}$.

Step 2. Let us now define the linear operator $\mathcal{K} \in \mathcal{L}\left(H_{\ell, 2}^{\alpha}\right)$ by

$$
\mathcal{K} w=3 u_{0}^{h} w .
$$

Then, the operator $\mathcal{A}+\mathcal{K}: H_{\ell, 2}^{1, \alpha} \rightarrow H_{\ell, 2}^{\alpha}$ is injective since it reduces to an injective operator when reduced on the real line (see [1]). So, to show that $\mathcal{A}+\mathcal{K}$ is an 
isomorphism of Banach spaces from $H_{\ell, 2}^{1, \alpha}$ onto $H_{\ell, 2}^{\alpha}$, it is sufficient to show that $\mathcal{A}^{-1} \mathcal{K}$ is a compact operator in $H_{\ell, 2}^{\alpha}$. To obtain this compactness it is sufficient to replace in the proof of Amick in [1] the interval of the real line by products of intervals with $]-\ell, \ell$, observing that our norm is a sup norm in the strip. The essential argument here is that $u_{0}^{h}$ converges uniformly to 0 when $|\mathcal{R} e(z)| \rightarrow \infty$ in the strip $\mathfrak{B}_{\ell}$, so the proof of Amick works on every horizontal line.

\subsection{Estimates of the nonlinear terms}

In this subsection we give estimates of the different terms involved in the four components of the nonlinear operator $\mathbf{G}$.

LEMmA 17. - There exists $c$ such that for $\left.\varepsilon \in] 0, \varepsilon_{0}\right], A_{0}^{\star} \in\left[-\delta_{2}, \delta_{2}\right], \varphi \in\left[-\varphi_{0}, \varphi_{0}\right]$ and for every $\mathfrak{h}_{1}=\left(q_{1}, q_{2}, w, Z\right)$ in $\mathfrak{B H}_{\ell}^{\alpha}\left(\frac{1}{2} \delta_{0}, \delta_{3}\right), \mathbf{G}\left(\mathfrak{h}_{1}, A_{0}^{\star}, \varphi, \varepsilon\right)$ lies in $H_{\ell, 2}^{\alpha} \times H_{\ell, 3}^{\alpha} \times$

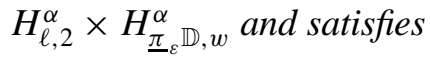

(i) $\left(\left\|G_{q_{1}}\right\|_{H_{\ell, 2}^{\alpha}}+\left\|G_{q_{2}}\right\|_{H_{\ell, 3}^{\alpha}}\right) \mathrm{e}^{-\frac{\lambda_{0} \ell}{\varepsilon}} \leqslant c\left(\varepsilon^{1-\alpha}+\left\|\mathfrak{h}_{1}\right\|_{\mathfrak{H}_{\ell}^{\alpha}}^{2}+\left|A_{0}^{\star}\right|\right)$,

(ii) $\left\|\left(G_{q_{2}}+i G_{q_{1}}\right) \mathrm{e}^{i \frac{\lambda_{0} z}{\varepsilon}}\right\|_{H_{\ell, 2}^{\alpha}}+\left\|\left(G_{q_{2}}-i G_{q_{1}}\right) \mathrm{e}^{-i \frac{\lambda_{0} z}{\varepsilon}}\right\|_{H_{\ell, 2}^{\alpha}} \leqslant c\left(\varepsilon^{1-\alpha}+\left\|\mathfrak{h}_{1}\right\|_{\mathfrak{H}_{\ell}^{\alpha}}^{2}+A_{0}^{\star}\right)$,

(iii) $\left\|\mathcal{G}_{w}\right\|_{H_{\ell, 2}^{\alpha}} \leqslant c\left(\varepsilon^{1-\alpha}\left(\varepsilon^{\alpha}+\left|A_{0}^{\star}\right|\right)+\left\|\mathfrak{h}_{1}\right\|_{\mathfrak{H}_{\ell}^{\alpha}}^{2}+\left|A_{0}^{\star}\right|\left\|\mathfrak{h}_{1}\right\|_{\mathfrak{H}_{\ell}^{\alpha}}\right)$,

(iv) $\left\|\mathcal{G}_{Z}\right\|_{H_{\underline{\pi}_{\varepsilon} \mathbb{D}, w}^{\alpha}} \leqslant c \varepsilon^{1-\alpha}\left(\varepsilon^{\alpha}+\left|A_{0}^{\star}\right|\right)$.

Proof. - This lemma readily follows from the following one observing that for $\mathfrak{h}_{1} \in$ $\mathfrak{B \mathfrak { H } _ { \ell } ^ { \alpha }}\left(\frac{1}{2} \delta_{0}, \delta_{3}\right), \mathfrak{h}=\mathfrak{h}_{0, \varepsilon}+\mathfrak{h}_{1}$ lies in $\mathfrak{B H}_{\ell}^{\alpha}\left(\delta_{0}, \delta_{3}\right)$ since $\left\|u_{0}^{h}\right\|_{H_{\ell, 2}^{1, \alpha}}+\left\|Y_{0}^{h}\right\|_{H_{\underline{\pi}_{\varepsilon}, w}^{\alpha}, w} \leqslant \frac{1}{2} \delta_{0}$ because of our choice of $\delta_{0}$ made in Definition 12 .

LEMMA 18. - There exists $c$ such that for $\left.\varepsilon \in] 0, \varepsilon_{0}\right], A_{0}^{\star} \in\left[-\delta_{2}, \delta_{2}\right], \varphi \in\left[-\varphi_{0}, \varphi_{0}\right]$ and for every $\mathfrak{h}=\left(q_{1}, q_{2}, u, Y\right)$ in $\mathfrak{B H}_{\ell}^{\alpha}\left(\delta_{0}, \delta_{3}\right)$

(i) $\left\|\Delta_{\gamma}^{\prime}\right\|_{H_{\ell, 2}^{\alpha}} \leqslant c \varepsilon$

(ii) $\left(\left\|R_{q_{1}}^{\prime}\right\|_{H_{\ell, 2}^{\alpha}}+\left\|R_{q_{2}}^{\prime}\right\|_{H_{\ell, 3}^{\alpha}}\right) \mathrm{e}^{-\frac{\lambda_{0} \ell}{\varepsilon}} \leqslant c \varepsilon^{1-\alpha}$,

(iii) $\left\|\left(i R_{q_{1}}^{\prime}+R_{q_{2}}^{\prime}\right) \mathrm{e}^{i \frac{\lambda_{0} z}{\varepsilon}}\right\|_{H_{\ell, 2}^{\alpha}}+\left\|\left(-i R_{q_{1}}^{\prime}+R_{q_{2}}^{\prime}\right) \mathrm{e}^{-i \frac{\lambda_{0} z}{\varepsilon}}\right\|_{H_{\ell, 2}^{\alpha}} \leqslant c \varepsilon^{1-\alpha}$,

(iv) $\left\|R_{\varepsilon, u}^{\prime}-R_{\varepsilon, u}\left(A_{0}^{2}\right)\right\|_{H_{\ell, 2}^{\alpha}} \leqslant c\left(\left\|\left(q_{1}, q_{2}\right)\right\|_{\mathfrak{Q}_{\ell}^{\alpha}}^{2}+2\left|A_{0}^{\star}\right|\left\|\left(q_{1}, q_{2}\right)\right\|_{\mathfrak{Q}_{\ell}^{\alpha}}\right)$,

(v) $\left\|\mathcal{R}_{\varepsilon, u}^{\prime}\right\|_{H_{\ell, 2}^{\alpha}}+\left\|\mathcal{R}_{\varepsilon, Y}^{\prime}\right\|_{H_{\underline{\underline{x}}_{\varepsilon} \mathbb{\mathbb { N }}, w}^{\alpha}} \leqslant c \varepsilon^{1-\alpha}\left(\varepsilon^{\alpha}+\left|A_{0}^{\star}\right|\right)$.

Proof. - (i) First observe that for every $\left.\left.\varphi \in\left[-\varphi_{0}, \varphi_{0}\right], \varepsilon \in\right] 0, \varepsilon_{0}\right], A_{0}^{\star} \in\left[-\delta_{2}, \delta_{2}\right]$, $z \in \mathfrak{B}_{\ell}$ and every $\left(q_{1}, q_{2}, u, Y\right)$ in $\mathfrak{B H}_{\ell}^{\alpha}\left(\delta_{0}, \delta_{3}\right)$,

$$
\begin{aligned}
& |u(z)|+\varepsilon|Y(z)|_{\widehat{\mathbb{\pi}_{\varepsilon} \mathbb{D}}} \leqslant\|u\|_{H_{\ell, 2}^{1, \alpha}}+\|Y\|_{H_{\mathbb{\pi}_{\varepsilon} \mathbb{D}, w}^{\alpha}}<\delta_{0}, \\
& |A(z)|=\left|\left(A_{0}+q_{2}(z)+i q_{1}(z)\right) \mathrm{e}^{i \psi_{\varphi}(z)}\right|<\delta_{1}, \\
& |\widetilde{A}(z)|=\left|\left(A_{0}+q_{2}(z)-i q_{1}(z)\right) \mathrm{e}^{-i \psi_{\varphi}(z)}\right|<\delta_{1}
\end{aligned}
$$


and

$$
\begin{aligned}
& \left\|\mathrm{e}^{ \pm i \psi_{\varphi}}\right\|_{C^{0}\left(\mathfrak{B}_{\ell}, \mathbb{C}\right)} \leqslant c \mathrm{e}^{\frac{\lambda_{0} \ell}{\varepsilon}}, \quad\left\|\mathrm{e}^{ \pm i \psi_{\varphi}}\right\|_{C^{\alpha}\left(\mathfrak{B}_{\ell}, \mathbb{C}\right)} \leqslant c \frac{\mathrm{e}^{\frac{\lambda_{0} \ell}{\varepsilon}}}{\varepsilon^{\alpha}}, \quad\left\|\mathrm{e}^{ \pm i\left(\psi_{\varphi}-\frac{\lambda_{0} z}{\varepsilon}\right)}\right\|_{C^{\alpha}\left(\mathfrak{B}_{\ell}, \mathbb{C}\right)} \leqslant c \\
& \|A\|_{C^{\alpha}\left(\mathfrak{B}_{\ell}, \mathbb{C}\right)} \leqslant c\left(\frac{\left|A_{0}^{\star}\right|}{\varepsilon^{\alpha}}+\left\|\left(q_{1}, q_{2}\right)\right\|_{\mathfrak{Q}_{\ell}^{\alpha}}\right) \\
& \|\widetilde{A}\|_{C^{\alpha}\left(\mathfrak{B}_{\ell}, \mathbb{C}\right)} \leqslant c\left(\frac{\left|A_{0}^{\star}\right|}{\varepsilon^{\alpha}}+\left\|\left(q_{1}, q_{2}\right)\right\|_{\mathfrak{Q}_{\ell}^{\alpha}}\right) \\
& \|A \widetilde{A}\|_{C^{\alpha}\left(\mathfrak{B}_{\ell}, \mathbb{C}\right)} \leqslant A_{0}^{2}+\left\|\left(q_{1}, q_{2}\right)\right\|_{\mathfrak{Q}_{\ell}^{\alpha}}^{2}+2\left|A_{0}^{\star}\right|\left\|\left(q_{1}, q_{2}\right)\right\|_{\mathfrak{Q}_{\ell}^{\alpha}} \\
& \text { and also } \quad\left\|p_{1}^{*}(Y)\right\|_{H_{\ell, 2}^{\alpha}} \leqslant\|Y\|_{H_{\mathbb{I}_{\varepsilon} \mathbb{D}, w}^{\alpha}}<\delta_{0} \\
& \|u\|_{H_{\ell, 2}^{\alpha}<\delta_{0},} \\
& \left\|A \widetilde{A}-A_{0}^{2}\right\|_{H_{\ell, 2}^{\alpha}} \leqslant\left\|\left(q_{1}, q_{2}\right)\right\|_{\mathfrak{Q}_{\ell}^{\alpha}}^{2}+2 \mid A_{0}^{\star}\left\|\left(q_{1}, q_{2}\right)\right\|_{\mathfrak{Q}_{\ell}^{\alpha}}
\end{aligned}
$$

since

$$
A \widetilde{A}-A_{0}^{2}=\left(\left(q_{2}+i q_{1}\right) \mathrm{e}^{i \frac{\lambda_{0} z}{\varepsilon}}\right)\left(\left(q_{2}-i q_{1}\right) \mathrm{e}^{-i \frac{\lambda_{0} z}{\varepsilon}}\right)+2 A_{0}^{\star} q_{2} \mathrm{e}^{-\frac{\lambda_{0} \ell}{\varepsilon}} .
$$

Estimate (i) follows from the above estimates combined with

$$
\begin{aligned}
& \left|\gamma_{1}\left(u_{*}, \varepsilon v_{*}, A_{*} \widetilde{A}_{*}, \varepsilon\right)-\gamma_{1}\left(0,0, A_{0}^{2}, \varepsilon\right)-\gamma_{1}\left(u_{*}, 0,0, \varepsilon\right)\right| \\
& \quad \leqslant c \varepsilon\left(\left|v_{*}\right|+\varepsilon\left|u_{*}\right| A_{0}^{2}+\left|A_{*} \widetilde{A}_{*}-A_{0}^{2}\right|\right)
\end{aligned}
$$

which holds for every $A_{*}, \widetilde{A}_{*}, u_{*}, v_{*} \in \mathbb{C}^{4}$ satisfying $\left|A_{*}\right|<\delta_{1},\left|\widetilde{A}_{*}\right|<\delta_{1},\left|u_{*}\right|<$ $\delta_{0},\left|v_{*}\right|<\delta_{0}$.

(ii) Estimates (34), (35-c, d), (36-a) combined with (15) ensures that

$$
\begin{aligned}
& \left\|R_{\varepsilon, A}^{\prime}\right\|_{C^{0}\left(\mathfrak{B}_{\ell}, \mathbb{C}\right)} \leqslant c \varepsilon\|Y\|_{H_{\underline{\pi}_{\varepsilon} \mathbb{\mathbb { D }}, w}^{\alpha}}\left(\|u\|_{H_{\ell, 2}^{1, \alpha}}+\|Y\|_{H_{\mathbb{\pi}_{\varepsilon} \mathbb{D}, w}^{\alpha}}\right) \leqslant c^{\prime} \varepsilon,
\end{aligned}
$$

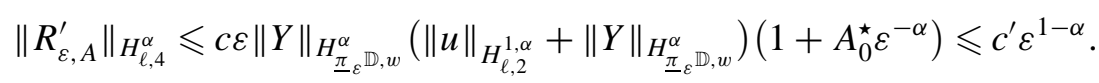

This two last estimates coupled with (i), (35-a) finally gives (ii).

(iii) The estimate (iii) can be deduced from the following explicit formulas

$$
\begin{aligned}
& \left(R_{q_{2}}^{\prime}+i R_{q_{1}}^{\prime}\right) \mathrm{e}^{i \frac{\lambda_{0} z}{\varepsilon}}=R_{\varepsilon, A}^{\prime} \mathrm{e}^{i\left(\frac{\lambda_{0} z}{\varepsilon}-\psi_{\varphi}\right)}+\left(q_{2}+i q_{1}\right) \mathrm{e}^{i \frac{\lambda_{0} z}{\varepsilon}} \Delta_{\gamma}^{\prime}+A_{0} \mathrm{e}^{i \frac{\lambda_{0} z}{\varepsilon}} \Delta_{\gamma}^{\prime}, \\
& \left(R_{q_{2}}^{\prime}-i R_{q_{1}}^{\prime}\right) \mathrm{e}^{-i \frac{\lambda_{0} z}{\varepsilon}}=R_{\varepsilon, \tilde{A}}^{\prime} \mathrm{e}^{i\left(\psi_{\varphi}-\frac{\lambda_{0} z}{\varepsilon}\right)}+\left(q_{2}-i q_{1}\right) \mathrm{e}^{-i \frac{\lambda_{0} z}{\varepsilon}} \Delta_{\gamma}^{\prime}+A_{0} \mathrm{e}^{-i \frac{\lambda_{0} z}{\varepsilon}} \Delta_{\gamma}^{\prime}
\end{aligned}
$$

combined with (i), (37-b) and (35-a, b).

(iv) Estimate (iv) directly follows from (34), (35-e), (36-b) and from the analyticity of $R_{\varepsilon, u}$.

(v) We deduce (v) from (13) and from (34), (35-c, d), (36-a).

We will also need estimates of the derivatives of $\mathbf{G}$ with respect to $\mathfrak{h}_{1}$. 
LEMMA 19. - There exists $c$ such that for $\left.\varepsilon \in] 0, \varepsilon_{0}\right]$ and every $\varphi \in\left[-\varphi_{0}, \varphi_{0}\right]$

(i) $\quad\left(\left\|D_{\mathfrak{h}_{1}} G_{q_{1}}(0,0, \varphi, \varepsilon)\right\|_{\mathcal{L}\left(\mathfrak{H}_{\ell}^{\alpha}, H_{\ell, 2}^{\alpha}\right)}+\left\|D_{\mathfrak{h}_{1}} G_{q_{2}}(0,0, \varphi, \varepsilon)\right\|_{\mathcal{L}\left(\mathfrak{H}_{\ell}^{\alpha}, H_{\ell, 3}^{\alpha}\right)}\right) \mathrm{e}^{-\frac{\lambda_{0} \ell}{\varepsilon}} \leqslant c \varepsilon^{1-\alpha}$,

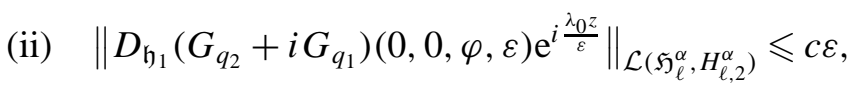

$$
\left\|D_{\mathfrak{h}_{1}}\left(G_{q_{2}}-i G_{q_{1}}\right)(0,0, \varphi, \varepsilon) \mathrm{e}^{-i \frac{\lambda_{0} z}{\varepsilon}}\right\|_{\mathcal{L}\left(\mathfrak{H}_{\ell}^{\alpha}, H_{\ell, 2}^{\alpha}\right)} \leqslant c \varepsilon
$$

(iii) $\left\|D_{\mathfrak{h}_{1}} \mathcal{G}_{w}(0,0, \varphi, \varepsilon)\right\|_{\mathcal{L}\left(\mathfrak{H}_{\ell}^{\alpha}, H_{\ell, 2}^{\alpha}\right)} \leqslant c \varepsilon$,

(iv) $\left\|D_{\mathfrak{h}_{1}} \mathcal{G}_{Z}(0,0, \varphi, \varepsilon)\right\|_{\left.\mathcal{L}_{\left(\mathfrak{H}_{\ell}, H_{\underline{\pi}_{\varepsilon}} \mathbb{D}, w\right.}^{\alpha}\right)} \leqslant c \varepsilon$.

Proof. - For $\mathfrak{h}^{\prime}=\left(q_{1}^{\prime}, q_{2}^{\prime}, w^{\prime}, Z^{\prime}\right) \in \mathfrak{H}_{\ell}^{\alpha}$ let us denote

$$
\mathrm{U}^{\prime}=\left(\left(q_{2}^{\prime}+i q_{1}^{\prime}\right) \mathrm{e}^{i \psi_{\varphi}},\left(q_{2}^{\prime}+i q_{1}^{\prime}\right) \mathrm{e}^{-i \psi_{\varphi}}, w^{\prime}, Z^{\prime}\right)
$$

and observe that

$$
\begin{aligned}
D_{\mathfrak{h}_{1}} G_{q_{1}}(0,0, \varphi, \varepsilon) \cdot \mathfrak{h}^{\prime}= & \frac{1}{2 i} \mathrm{e}^{-i \psi_{\varphi}} D_{\cup} R_{\varepsilon, A}\left(0,0, u_{0}^{h}, Y_{0}^{h}\right) \cdot \mathrm{U}^{\prime} \\
& -\frac{1}{2 i} \mathrm{e}^{i \psi_{\varphi}} D_{\cup} R_{\varepsilon, \tilde{A}}\left(0,0, u_{0}^{h}, Y_{0}^{h}\right) \cdot \mathrm{U}^{\prime} \\
& +q_{2}^{\prime}\left(\gamma_{1}\left(u_{0}^{h}, \varepsilon p_{1}^{*}\left(Y_{0}^{h}\right), 0, \varepsilon\right)-\gamma_{1}\left(u_{0}^{h}, 0,0, \varepsilon\right)\right), \\
D_{\mathfrak{h}_{1}} G_{q_{2}}(0,0, \varphi, \varepsilon) \cdot \mathfrak{h}^{\prime}= & \frac{1}{2} \mathrm{e}^{-i \psi_{\varphi}} D_{\cup} R_{\varepsilon, A}\left(0,0, u_{0}^{h}, Y_{0}^{h}\right) \cdot \mathrm{U}^{\prime} \\
& +\frac{1}{2} \mathrm{e}^{i \psi_{\varphi}} D_{\cup} R_{\varepsilon, \tilde{A}}\left(0,0, u_{0}^{h}, Y_{0}^{h}\right) \cdot \mathrm{U}^{\prime} \\
& -q_{1}^{\prime}\left(\gamma_{1}\left(u_{0}^{h}, \varepsilon p_{1}^{*}\left(Y_{0}^{h}\right), 0, \varepsilon\right)-\gamma_{1}\left(u_{0}^{h}, 0,0, \varepsilon\right)\right), \\
D_{\mathfrak{h}_{1}} \mathcal{G}_{w}(0,0, \varphi, \varepsilon) \cdot \mathfrak{h}^{\prime}= & D_{\cup} \mathcal{R}_{\varepsilon, u}\left(0,0, u_{0}^{h}, Y_{0}^{h}\right) \cdot \mathrm{U}^{\prime}, \\
D_{\mathfrak{h}_{1}} \mathcal{G}_{Z}(0,0, \varphi, \varepsilon) \cdot \mathfrak{h}^{\prime}= & D_{\cup} \mathcal{R}_{\varepsilon, Y}\left(0,0, u_{0}^{h}, Y_{0}^{h}\right) \cdot \mathrm{U}^{\prime} .
\end{aligned}
$$

Then observing that (35-b) and our choice of $\delta_{0}$ ensure respectively that

$$
\left\|\mathrm{U}^{\prime}\right\|_{\mathrm{E}_{\ell}^{\alpha}} \leqslant c\left\|\mathfrak{h}^{\prime}\right\|_{\mathfrak{H}_{\ell}^{\alpha}}, \quad\left|u_{0}^{h}\right|+\varepsilon\left|Y_{0}^{h}\right| \leqslant\left\|u_{0}^{h}\right\|_{H_{\ell, 2}^{1, \alpha}}+\left\|Y_{0}^{h}\right\|_{H_{\underline{\underline{x}}_{\varepsilon} \mathbb{\mathbb { N }}, w}^{\alpha}}<\delta_{0},
$$

estimates (iii) and (iv) directly follows from (40) and (14).

To get (i), we first check that:

(a) the analyticity of $R_{\varepsilon, A}, R_{\varepsilon, \tilde{A}}$ combined with (15) ensures that

$$
\left|D_{\cup} R_{\varepsilon, A}\left(0,0, u_{0}^{h}, Y_{0}^{h}\right)\right|_{\mathcal{L}\left(\mathrm{E}_{\ell}^{\alpha}, H_{\ell, 4}^{\alpha}\right)}+\left|D_{\cup} R_{\varepsilon, \tilde{A}}\left(0,0, u_{0}^{h}, Y_{0}^{h}\right)\right|_{\mathcal{L}\left(\mathrm{E}_{\ell}^{\alpha}, H_{\ell, 4}^{\alpha}\right)} \leqslant c \varepsilon ;
$$

(b) the explicit formula giving $\gamma_{1}$ at Lemma 3 combined with (36-a) ensure that

$$
\left\|\gamma_{1}\left(u_{0}^{h}, \varepsilon p_{1}^{*}\left(Y_{0}^{h}\right), 0, \varepsilon\right)-\gamma_{1}\left(u_{0}^{h}, 0,0, \varepsilon\right)\right\|_{H_{\ell, 2}^{\alpha}} \leqslant c \varepsilon .
$$


We deduce estimate (i) from (35-a) and the two above estimates.

Finally, (ii) follows from the above estimates, (35-b) and from the explicit formulas

$$
\begin{aligned}
\mathrm{e}^{i \frac{\lambda_{0} z}{\varepsilon}} & D_{\mathfrak{h}_{1}}\left(G_{q_{2}}+i G_{q_{1}}\right)(0,0, \varphi, \varepsilon) \cdot \mathrm{U}^{\prime} \\
= & \mathrm{e}^{i\left(\frac{\lambda_{0} z}{\varepsilon}-\psi_{\varphi}\right)} D_{\cup} R_{\varepsilon, A}\left(0,0, u_{0}^{h}, Y_{0}^{h}\right) \cdot \mathrm{U}^{\prime} \\
& +i\left(q_{2}^{\prime}+i q_{1}^{\prime}\right) \mathrm{e}^{i \frac{\lambda_{0} z}{\varepsilon}}\left(\gamma_{1}\left(u_{0}^{h}, \varepsilon p_{1}^{*}\left(Y_{0}^{h}\right), 0, \varepsilon\right)-\gamma_{1}\left(u_{0}^{h}, 0,0, \varepsilon\right)\right), \\
\mathrm{e}^{-i \frac{\lambda_{0} z}{\varepsilon}} D_{\mathfrak{h}_{1}}\left(G_{q_{2}}-i G_{q_{1}}\right)(0,0, \varphi, \varepsilon) \cdot \mathrm{U}^{\prime} & \\
= & e^{i\left(\psi_{\varphi}-\frac{\lambda_{0} z}{\varepsilon}\right)} D_{\cup} R_{\varepsilon, \tilde{A}}\left(0,0, u_{0}^{h}, Y_{0}^{h}\right) \cdot \mathrm{U}^{\prime} \\
& +i\left(q_{2}^{\prime}-i q_{1}^{\prime}\right) \mathrm{e}^{-i \frac{\lambda_{0} z}{\varepsilon}}\left(\gamma_{1}\left(u_{0}^{h}, \varepsilon p_{1}^{*}\left(Y_{0}^{h}\right), 0, \varepsilon\right)-\gamma_{1}\left(u_{0}^{h}, 0,0, \varepsilon\right)\right) .
\end{aligned}
$$

\subsection{Splitting of the solvability condition}

This subsection is devoted to the computation of an equivalent of the solvability condition $J$. On one hand, the leading part of $J$ is given by an explicit computation involving only $\mathfrak{h}_{0, \varepsilon}$. On the other hand, the perturbation term of $J$ which involves the perturbation part of the homoclinic happens to be an oscillatory integral which admits an exponentially small upper bound given by the Exponential Lemma 4. We have complexified the problem to be able to obtain this exponentially small upper bound.

LEMMA 20. - There exists $c$ such that for every $\left.\left.\varphi \in\left[-\varphi_{0}, \varphi_{0}\right], \varepsilon \in\right] 0, \varepsilon_{0}\right], A_{0}^{\star} \in$ $\left[-\delta_{2}, \delta_{2}\right]$ and every reversible $\mathfrak{h}_{1}=\left(q_{1}, q_{2}, w, Z\right) \in \mathfrak{B H}_{\ell}^{\alpha}\left(\frac{1}{2} \delta_{0}, \delta_{3}\right)$,

$$
J\left(\mathfrak{h}_{1}, \varphi, A_{0}^{\star}, \varepsilon\right)=\mathrm{e}^{\frac{-\ell \lambda_{0}}{\varepsilon}} A_{0}^{\star} \sin (\Gamma(\infty))+J_{1}\left(\mathfrak{h}_{1}, \varphi, A_{0}^{\star}, \varepsilon\right)
$$

with

$$
\Gamma(\infty)=\int_{0}^{+\infty} \gamma_{1}\left(u_{0}^{h}(\underline{x}), 0,0, \varepsilon\right) d \underline{x}-\varphi \frac{\rho \pi}{2}\left(\lambda_{\varepsilon}+\varepsilon \gamma_{1}\left(0,0, A_{0}^{2}, \varepsilon\right)\right)
$$

and

(i) $\left|J_{1}\left(\mathfrak{h}_{1}, \varphi, A_{0}^{\star}, \varepsilon\right)\right| \leqslant c \mathrm{e}^{\frac{-\ell \lambda_{0}}{\varepsilon}}\left(\varepsilon+\left|A_{0}^{\star}\right|\left\|\mathfrak{h}_{1}\right\|_{\mathfrak{H}_{\ell}^{\alpha}}+\left\|\mathfrak{h}_{1}\right\|_{\mathfrak{H}_{\ell}^{\alpha}}^{2}\right)$,

(ii) $\left\|D_{\mathfrak{h}_{1}} J_{1}(0, \varphi, 0, \varepsilon)\right\|_{\mathcal{L}\left(\mathfrak{H}_{\ell}^{\alpha}, \mathbb{R}\right)} \leqslant c \varepsilon \mathrm{e}^{\frac{-\ell \lambda_{0}}{\varepsilon}}$.

Proof. - (i) Using the reversibility of $\mathfrak{h}_{1}$ and the antireversiblity of $\mathbf{G}$ we get

$$
J=\int_{0}^{\infty}\left\langle r_{-}, \mathbf{G}\left(\mathfrak{h}_{1}, A_{0}^{\star}, \varphi, \varepsilon\right)\right\rangle_{*} d \underline{x}=\frac{1}{2 i} \int_{-\infty}^{+\infty} \mathrm{e}^{-i \Gamma}\left(i G_{q_{1}}+G_{q_{2}}\right)\left(\mathfrak{h}_{1}, A_{0}^{\star}, \varphi, \varepsilon\right) d \underline{x} .
$$

Moreover,

$$
\begin{aligned}
i G_{q_{1}}+G_{q_{2}} & =i R_{q_{1}}^{\prime}+R_{q_{2}}^{\prime}+i\left(i q_{1}+q_{2}\right) \Delta_{\gamma}^{h}+i A_{0}\left(\gamma_{1}\left(u_{0}^{h}+w, 0,0, \varepsilon\right)-\varphi \rho_{0}\right) \\
& =i A_{0}\left(\gamma_{1}\left(u_{0}^{h}, 0,0, \varepsilon\right)-\varphi \rho_{0}\right)+i A_{0}\left(\Delta_{\gamma}^{h}+\Delta_{\gamma}^{\prime}\right)+\mathrm{e}^{-i \frac{\lambda_{0} z}{\varepsilon}} \Delta_{\varepsilon, A}
\end{aligned}
$$


where

$$
\begin{aligned}
\Delta_{\varepsilon, A} & =R_{\varepsilon, A}^{\prime} \mathrm{e}^{i\left(\frac{\lambda_{0} z}{\varepsilon}-\psi_{\varphi}\right)}+i\left(\Delta_{\gamma}^{h}+\Delta_{\gamma}^{\prime}\right)\left(i q_{1}+q_{2}\right) \mathrm{e}^{i \frac{\lambda_{0} z}{\varepsilon}} \\
\Delta_{\gamma}^{h} & =\left(\gamma_{1}\left(u_{0}^{h}+w, 0,0, \varepsilon\right)-\gamma_{1}\left(u_{0}^{h}, 0,0, \varepsilon\right)\right) .
\end{aligned}
$$

So we split $J$ in three parts. The first one gives the leading part of $J$ :

$$
\begin{aligned}
J_{0} & =\frac{1}{2 i} \int_{-\infty}^{+\infty} \mathrm{e}^{-i \Gamma} i A_{0}\left(\gamma_{1}\left(u_{0}^{h}, 0,0, \varepsilon\right)-\varphi \rho_{0}\right) d \underline{x} \\
& =A_{0} \int_{0}^{+\infty} \cos (\Gamma) \frac{d \Gamma}{d \underline{x}} d \underline{x}=A_{0} \sin (\Gamma(\infty)) .
\end{aligned}
$$

The second one can be bounded using Lemma 18(i). We get that

$$
\begin{aligned}
\left|J_{10}\right|=\left|\frac{1}{2 i} \int_{-\infty}^{+\infty} \mathrm{e}^{-i \Gamma} i A_{0}\left(\Delta_{\gamma}^{h}+\Delta_{\gamma}^{\prime}\right) d \underline{x}\right| & \leqslant c\left|A_{0}\right|\left(\|w\|_{H_{\ell, 2}^{1, \alpha}}+\varepsilon\right) \\
& \leqslant c\left|A_{0}\right|\left(\left\|\mathfrak{h}_{1}\right\|_{\mathfrak{H}_{\ell}^{\alpha}}+\varepsilon\right) .
\end{aligned}
$$

The third part of $J$ happens to be an oscillatory integral given by

$$
J_{11}=\frac{1}{2 i} \int_{-\infty}^{+\infty} \mathrm{e}^{-i \frac{\lambda_{0} \underline{\underline{x}}}{\varepsilon}} \mathrm{e}^{-i \Gamma} \Delta_{\varepsilon, A} d \underline{x} .
$$

Observe that

$$
\sup _{\substack{z \in \mathfrak{B}_{\ell} \\ A_{0}^{\star} \in\left[-\delta_{2}, \delta_{2}\right], \varepsilon \in\left[0, \varepsilon_{0}\right]}}|\mathcal{I} m(\Gamma(z))|+\left|\mathcal{I} m\left(\frac{\lambda_{0} z}{\varepsilon}-\psi_{\varphi}\right)\right|<\infty
$$

and that for every $\left.\left.\varphi \in\left[-\varphi_{0}, \varphi_{0}\right], \varepsilon \in\right] 0, \varepsilon_{0}\right], A_{0}^{\star} \in\left[-\delta_{2}, \delta_{2}\right], z \in \mathfrak{B}_{\ell}$ and every $\mathfrak{h}_{1} \in$ $\mathfrak{B H}_{\ell}^{\alpha}\left(\frac{1}{2} \delta_{0}, \delta_{3}\right)$,

$$
\begin{aligned}
& \left|u_{0}^{h}(z)+w(z)\right|+\varepsilon\left|Y_{0}^{h}(z)+Z(z)\right|_{\widehat{\pi_{\varepsilon} \mathbb{D}}}<\delta_{0}, \\
& |A(z)|=\left|\left(A_{0}+q_{2}(z)+i q_{1}(z)\right) \mathrm{e}^{i \psi_{\varphi}(z)}\right|<\delta_{1}, \\
& |\widetilde{A}(z)|=\left|\left(A_{0}+q_{2}(z)-i q_{1}(z)\right) \mathrm{e}^{-i \psi_{\varphi}(z)}\right|<\delta_{1} .
\end{aligned}
$$

Hence, using (46), (15) and Lemma 18(i), we get that there exists $c$ such that

$$
\begin{aligned}
\sup _{z \in \mathfrak{B}_{\ell}}\left(1+|z|^{2}\right)\left|\mathrm{e}^{-i \Gamma(z)} \Delta_{\varepsilon, A}(z)\right| \leqslant & c \varepsilon\left\|Y_{0}^{h}+Z\right\|_{H_{\boldsymbol{\pi}_{\varepsilon} \mathbb{D}, w}^{\alpha}}\left(\left\|u_{0}^{h}+w\right\|_{H_{\ell, 2}^{1, \alpha}}+\left\|Y_{0}^{h}+Z\right\|_{H_{\boldsymbol{\pi}_{\varepsilon} \mathbb{D}, w}^{\alpha}}\right) \\
& +c\left(\|w\|_{H_{\ell, 2}^{1, \alpha}}+\varepsilon\right)\left\|\left(q_{1}, q_{2}\right)\right\|_{\mathfrak{Q}_{\ell}^{\alpha}} \\
\leqslant & \varepsilon\left(2 c \delta_{0}^{2}+c \delta_{3}\right)+c\left\|\mathfrak{h}_{1}\right\|_{\mathfrak{H}_{\ell}^{\alpha}}^{2}
\end{aligned}
$$

for every $\left.\left.\varphi \in\left[-\varphi_{0}, \varphi_{0}\right], \varepsilon \in\right] 0, \varepsilon_{0}\right], A_{0}^{\star} \in\left[-\delta_{2}, \delta_{2}\right]$ and every $\mathfrak{h}_{1} \in \mathfrak{B H}_{\ell}^{\alpha}\left(\frac{1}{2} \delta_{0}, \delta_{3}\right)$. 
This last estimate combined with the Exponential Lemma 4 ensures that there exists $c$ such that

$$
\left|J_{11}\right| \leqslant c \mathrm{e}^{-\frac{\lambda_{0} \ell}{\varepsilon}}\left(\varepsilon+\left\|\mathfrak{h}_{1}\right\|_{\mathfrak{H}_{\ell}^{\alpha}}^{2}\right)
$$

holds for for every $\left.\left.\varphi \in\left[-\varphi_{0}, \varphi_{0}\right], \varepsilon \in\right] 0, \varepsilon_{0}\right], A_{0}^{\star} \in\left[-\delta_{2}, \delta_{2}\right]$ and every $\mathfrak{h}_{1} \in \mathfrak{B H}_{\ell}^{\alpha}\left(\frac{1}{2} \delta_{0}, \delta_{3}\right)$.

Gathering (44), (45), (47), we finally get the desired equivalent of $J$ at Lemma 20(i).

(ii) For $\mathfrak{h}^{\prime}=\left(q_{1}^{\prime}, q_{2}^{\prime}, w^{\prime}, Z^{\prime}\right) \in \mathfrak{H}_{\ell}^{\alpha}$ let us denote

$$
\mathrm{U}^{\prime}=\left(\left(q_{2}^{\prime}+i q_{1}^{\prime}\right) \mathrm{e}^{i \psi_{\varphi}},\left(q_{2}^{\prime}+i q_{1}^{\prime}\right) \mathrm{e}^{-i \psi_{\varphi}}, w^{\prime}, Z^{\prime}\right)
$$

and observe that

$$
\begin{aligned}
D_{\mathfrak{h}_{1}} J_{1}(0, \varphi, 0, \varepsilon) \cdot \mathfrak{h}^{\prime} & =D_{\mathfrak{h}_{1}} J_{11}(0, \varphi, 0, \varepsilon) \cdot \mathfrak{h}^{\prime} \\
& =\frac{1}{2 i} \int_{-\infty}^{+\infty} \mathrm{e}^{-i \frac{\lambda_{0} \underline{\underline{x}}}{\varepsilon}} \mathrm{e}^{-i \Gamma} D_{\mathfrak{h}_{1}} \Delta_{\varepsilon, A}(0, \varphi, 0, \varepsilon) \cdot \mathfrak{h}^{\prime} d \underline{x}
\end{aligned}
$$

with

$$
\begin{aligned}
D_{\mathfrak{h}_{1}} \Delta_{\varepsilon, A}(0, \varphi, 0, \varepsilon) \cdot \mathfrak{h}^{\prime}= & \mathrm{e}^{i\left(\frac{\lambda_{0} z}{\varepsilon}-\psi_{\varphi}\right)} D_{\cup} R_{\varepsilon, A}\left(0,0, u_{0}^{h}, Y_{0}^{h}\right) \cdot \mathrm{U}^{\prime} \\
& +i\left(q_{2}^{\prime}+i q_{1}^{\prime}\right) \mathrm{e}^{i \frac{\lambda_{0} z}{\varepsilon}}\left(\gamma_{1}\left(u_{0}^{h}, \varepsilon p_{1}^{*}\left(Y_{0}^{h}\right), 0, \varepsilon\right)-\gamma_{1}\left(u_{0}^{h}, 0,0, \varepsilon\right)\right) .
\end{aligned}
$$

Hence, using (46), (41), (42), (43) we get

$$
\sup _{z \in \mathfrak{B}_{\ell}}\left(1+|z|^{2}\right)\left|\mathrm{e}^{-i \Gamma(z)} D_{\mathfrak{h}_{1}} \Delta_{\varepsilon, A}(0, \varphi, 0, \varepsilon) \cdot \mathfrak{h}^{\prime}(z)\right| \leqslant c \varepsilon\left\|\mathfrak{h}^{\prime}\right\|_{\mathfrak{H}_{\ell}^{\alpha}} .
$$

This last estimate combined with the Exponential Lemma 4 ensures that there exists $c$ such that

$$
\left|D_{\mathfrak{h}_{1}} J_{1}(0, \varphi, 0, \varepsilon) \cdot \mathfrak{h}^{\prime}\right| \leqslant c \varepsilon \mathrm{e}^{\frac{-\ell \lambda_{0}}{\varepsilon}}\left\|\mathfrak{h}^{\prime}\right\|_{\mathfrak{H}_{\ell}^{\alpha}}
$$

holds for for every $\left.\left.\varphi \in\left[-\varphi_{0}, \varphi_{0}\right], \varepsilon \in\right] 0, \varepsilon_{0}\right]$ and every $\mathfrak{h}^{\prime} \in \mathfrak{H}_{\ell}^{\alpha}$.

\subsection{Proof of Theorem 9}

\subsubsection{Homoclinic connections of the modified equations}

As already explained in subsection 4.2, for finding homoclinic connections of (20) we first study the modified Eq. (22)

$$
\mathbf{L}_{\varphi}(z) \mathfrak{h}_{1}=\mathbf{G}^{\perp}\left(\mathfrak{h}_{1}, A_{0}^{\star}, \varphi, \varepsilon\right),
$$

where

$$
\mathbf{G}^{\perp}=\mathbf{G}-\frac{2}{\sqrt{\pi}} J \mathrm{e}^{-z^{2}} r_{-} .
$$

We first prove

Proposition 21. - For every $\ell \in] 0, \rho\left[, 0<\alpha<1\right.$, there exist $\delta_{4}, \varepsilon_{1}$ and $c>0$ such that for every $\left.\varphi \in]-\varphi_{0}, \varphi_{0}[, \varepsilon \in] 0, \varepsilon_{1}\right]$, and every $\left.A_{0}^{\star} \in\right]-\delta_{4}, \delta_{4}[$, Eq. (22) admits 
a reversible homoclinic connection $\mathfrak{h}_{1, A_{0}^{\star}, \varphi, \varepsilon}$ to 0 , lying in $\mathfrak{H}_{\ell}^{\alpha}$, analytic with respect to $\left(\varphi, A_{0}^{\star}\right)$ and satisfying

$$
\left\|\mathfrak{h}_{1}\right\|_{\mathfrak{H}_{\ell}^{\alpha}} \leqslant c\left(\varepsilon^{1-\alpha}+\left|A_{0}^{\star}\right|\right) .
$$

Proof. - Our aim is to solve (22) by using analytic implicit function theorem. For that purpose, we rewrite (22) under a more appropriate form.

On one hand, Lemmas 15 and 16 ensure that for every $\varphi \in\left[-\varphi_{0}, \varphi_{0}\right]$ and every $\left.\varepsilon \in] 0, \varepsilon_{0}\right]$, the operator $L_{\varphi}$ is an isomorphism of Banach spaces from

$$
\left.\mathfrak{H}_{\ell}^{\alpha}\right|_{R}:=\mathfrak{H}_{\ell}^{\alpha} \cap\left\{\mathfrak{h} / \mathfrak{h} \text { is reversible, i.e. } \mathfrak{S h}(z)=\mathfrak{h}(-z) \text { for any } z \in \mathfrak{B}_{\ell}\right\}
$$

onto

$$
\left.\mathbf{H}_{\ell}^{\alpha}\right|_{A R} ^{\perp}:=\left(H_{\ell, 2}^{\alpha} \times H_{\ell, 3}^{\alpha} \times H_{\ell, 2}^{\alpha} \times H_{\underline{\pi}_{\varepsilon} \mathbb{D}, w}^{\alpha}\right) \cap\left\{\mathbf{F} / \int_{0}^{\infty}\left\langle r_{-}(\underline{x}), \mathbf{F}(\underline{x})\right\rangle d \underline{x}=0\right\}
$$

$\cap\left\{\mathbf{F} / \mathbf{F}\right.$ is antireversible, i.e. $\mathfrak{S} \mathbf{F}(z)=-\mathbf{F}(-z)$ for any $\left.z \in \mathfrak{B}_{\ell}\right\}$

normed with

$$
\begin{aligned}
\|\mathbf{F}\|_{\mathbf{H}_{\ell}^{\alpha}}:= & \left\|F_{w}\right\|_{H_{\ell, 2}^{\alpha}}+\left\|F_{Z}\right\|_{H_{\mathbb{I}_{\varepsilon}}^{\alpha} \mathbb{\mathbb { D }}, w}+\mathrm{e}^{-\frac{\lambda_{0} \ell}{\varepsilon}}\left(\left\|F_{q_{1}}\right\|_{H_{\ell, 2}^{\alpha}}+\left\|F_{q_{2}}\right\|_{H_{\ell, 3}^{\alpha}}\right) \\
& +\left\|\left(i F_{q_{1}}+F_{q_{2}}\right) \mathrm{e}^{i \frac{\lambda_{0} z}{\varepsilon}}\right\|_{H_{\ell, 2}^{\alpha}}+\left\|\left(-i F_{q_{1}}+F_{q_{2}}\right) \mathrm{e}^{-i \frac{\lambda_{0} z}{\varepsilon}}\right\|_{H_{\ell, 2}^{\alpha}},
\end{aligned}
$$

where $\mathbf{F}=\left(F_{q_{1}}, F_{q_{2}}, F_{w}, F_{Z}\right)$.

On the other hand, Lemmas 17 and 20 ensure that $\mathbf{G}^{\perp}\left(\mathfrak{h}_{1}, A_{0}^{\star}, \varphi, \varepsilon\right)$ lies in $\left.\mathbf{H}_{\ell}^{\alpha}\right|_{A R} ^{\perp}$ for every $\left.\left.\varphi \in\left[-\varphi_{0}, \varphi_{0}\right], \varepsilon \in\right] 0, \varepsilon_{0}\right], A_{0}^{\star} \in\left[-\delta_{2}, \delta_{2}\right]$ and every $\left.\mathfrak{h}_{1} \in \mathfrak{B} \mathfrak{H}_{\ell}^{\alpha}\left(\frac{1}{2} \delta_{0}, \delta_{3}\right)\right|_{R}$ where $\left.\mathfrak{B H}_{\ell}^{\alpha}\left(d_{0}, d_{1}\right)\right|_{R}=\mathfrak{B H}_{\ell}^{\alpha}\left(d_{0}, d_{1}\right) \cap\{\mathfrak{h} / \mathfrak{h}$ is reversible $\}$.

Hence, for every $\left.\left.\varphi \in\left[-\varphi_{0}, \varphi_{0}\right], \varepsilon \in\right] 0, \varepsilon_{0}\right], A_{0}^{\star} \in\left[-\delta_{2}, \delta_{2}\right]$ and every $\mathfrak{h}_{1} \in \mathfrak{B} \mathfrak{H}_{\ell}^{\alpha}\left(\frac{1}{2} \delta_{0}\right.$, $\left.\delta_{3}\right)\left.\right|_{R}$ Eq. (22) is equivalent to

$$
\mathfrak{h}_{1}=\mathbf{N}_{\varepsilon}^{\perp}\left(\mathfrak{h}_{1}, A_{0}^{\star}, \varphi\right):=\mathbf{L}_{\varphi}^{-1} \mathbf{G}^{\perp}\left(\mathfrak{h}_{1}, A_{0}^{\star}, \varphi, \varepsilon\right)
$$

where $\mathbf{N}_{\varepsilon}^{\perp}$ is an analytic function from $\left.\left.\mathfrak{B H}_{\ell}^{\alpha}\left(\frac{1}{2} \delta_{0}, \delta_{3}\right)\right|_{R} \times\right]-\delta_{2}, \delta_{2}[\times]-\varphi_{0}, \varphi_{0}\left[\right.$ to $\left.\mathfrak{H}_{\ell}^{\alpha}\right|_{R}$ which satisfies

$$
\begin{aligned}
& \left\|\mathbf{N}_{\varepsilon}^{\perp}\left(\mathfrak{h}_{1}, A_{0}^{\star}, \varphi\right)\right\|_{\mathfrak{H}_{\ell}^{\alpha}} \leqslant c\left(\varepsilon^{1-\alpha}+\left\|\mathfrak{h}_{1}\right\|_{\mathfrak{H}_{\ell}^{\alpha}}^{2}+\left|A_{0}^{\star}\right|\right), \\
& \left\|D_{\mathfrak{h}_{1}} \mathbf{N}_{\varepsilon}^{\perp}(0,0, \varphi)\right\|_{\mathcal{L}\left(\mathfrak{H}_{\ell}^{\alpha}, \mathfrak{H}_{\ell}^{\alpha}\right)} \leqslant c \varepsilon^{1-\alpha}
\end{aligned}
$$

thanks to Lemmas $15,16,17,19,20$ and thanks to the estimate

$$
\left\|\mathrm{e}^{-z^{2}} r_{-}\right\|_{\mathbf{H}_{\ell}^{\alpha}} \leqslant c \varepsilon^{-\alpha} \mathrm{e}^{\frac{\lambda_{0} \ell}{\varepsilon}}
$$

Finally, we need a slight adaptation of the Implicit Function Theorem to solve (48), since we fix $\varepsilon$ small enough, but non zero here. We replace $\mathbf{N}_{\varepsilon}^{\perp}\left(\mathfrak{h}_{1}, A_{0}^{\star}, \varphi\right)$ by

$$
\mathbf{N}_{\varepsilon}^{\perp}\left(\mathfrak{h}_{1}, A_{0}^{\star}, \varphi\right)-\left(1-\mu \varepsilon^{\alpha-1}\right) \mathbf{N}_{\varepsilon}^{\perp}(0,0, \varphi)
$$


and use the Analytic Implicit Function Theorem for $\left(\mathfrak{h}_{1}, A_{0}^{\star}, \mu\right)$ near 0 , observing that $\varepsilon^{\alpha-1} \mathbf{N}_{\varepsilon}^{\perp}(0,0, \varphi)$ is uniformly bounded in $\left.\mathfrak{H}_{\ell}^{\alpha}\right|_{R}$ for $\left.\left.\left.(\varepsilon, \varphi) \in\right] 0, \varepsilon\right] \times\right] \varphi_{0}, \varphi_{0}[$.

For any $\varphi \in] \varphi_{0}, \varphi_{0}\left[\right.$ and for $\mu=0$ we have the trivial solution $\left(\mathfrak{h}_{1}, A_{0}^{\star}\right)=0$ whereas (48) corresponds to $\mu=\varepsilon^{1-\alpha}$ which lies in the domain of existence of the solution for $\varepsilon$ and $\left|A_{0}^{\star}\right|$ small enough.

Hence, there exist $\varepsilon_{1}, \delta_{4}$ such that for every $\left.\left.\varepsilon \in\right] 0, \varepsilon_{1}\right],(22)$ admits a solution $\mathfrak{h}_{1, \varphi, A_{0}^{\star}, \varepsilon}$ lying in $\left.\mathfrak{H}_{\ell}^{\alpha}\right|_{R}$ which is analytic with respect to $\left.\left(\varphi, A_{0}^{\star}\right) \in\right]-\varphi_{0}, \varphi_{0}[\times]-\delta_{4}, \delta_{4}[$ and which satisfies

$$
\left\|\mathfrak{h}_{1, \varphi, A_{0}^{\star}, \varepsilon}\right\|_{\mathfrak{H}_{\ell}^{\alpha}} \leqslant c\left(\varepsilon^{1-\alpha}+\left|A_{0}^{\star}\right|\right) .
$$

\subsubsection{Resolution of the solvability condition}

To achieve the proof of Theorem 9, we look for appropriate values of the phase shift $\left.\varphi:=\varphi\left(A_{0}^{\star}, \varepsilon\right) \in\right]-\varphi_{0}, \varphi_{0}[$ such that

$$
J\left[\mathfrak{h}_{1, A_{0}^{\star}, \varphi\left(\varepsilon, A_{0}^{\star}\right), \varepsilon}, \varphi\left(\varepsilon, A_{0}^{\star}\right), A_{0}^{\star}, \varepsilon\right]=0 .
$$

For such values of the phase shift, $\mathfrak{h}_{1, A_{0}^{\star}, \varphi\left(\varepsilon, A_{0}^{\star}\right), \varepsilon}$ is a reversible homoclinic connection to 0 of the Eq. (20) lying in $\mathfrak{B H}_{\ell}^{\alpha}\left(\frac{1}{2} \delta_{0}, \delta_{3}\right)$ which gives the existence of an homoclinic connection to 0 for (19) under the form

$$
\mathfrak{h}=\mathfrak{h}_{0, \varepsilon}+\mathfrak{h}_{1, A_{0}^{\star}, \varphi\left(\varepsilon, A_{0}^{\star}\right), \varepsilon}
$$

Denote $\varepsilon_{2}:=\min \left(\varepsilon_{1}, \delta_{4}^{2}\right)$. Then Proposition 21 and Lemma 20 ensure that there exists $c_{2}>0$ such that for any $\left.\left.\alpha \in\right] 0, \frac{1}{2}\right]$, every $\left.\varepsilon \in\right] 0, \varepsilon_{2}[, \varphi \in]-\varphi_{0}, \varphi_{0}[$ and every $A_{0}^{\star} \in\left[0, \varepsilon^{1-\alpha}\right]$, we have $\varepsilon^{1-\alpha}<\delta_{4}$ and

$$
J\left(\mathfrak{h}_{1, A_{0}^{\star}, \varphi, \varepsilon}, \varphi, A_{0}^{\star}, \varepsilon\right)=\mathrm{e}^{\frac{-\ell \lambda_{0}}{\varepsilon}} A_{0}^{\star} \sin \left(\Gamma_{\varphi}(\infty)\right)+J_{1}\left(\mathfrak{h}_{1, A_{0}^{\star}, \varphi, \varepsilon}, \varphi, A_{0}^{\star}, \varepsilon\right)
$$

and

$$
\left|J_{1}\left(\mathfrak{h}_{1, A_{0}^{\star}, \varphi, \varepsilon}, \varphi, A_{0}^{\star}, \varepsilon\right)\right| \leqslant c_{2} \varepsilon \mathrm{e}^{\frac{-\ell \lambda_{0}}{\varepsilon}} .
$$

Then observe that $\varphi \mapsto \Gamma_{\varphi}(\infty)$ is affine since $\Gamma_{\varphi}(\infty)=a\left(\varepsilon, A_{0}^{\star}\right) \varphi+b(\varepsilon)$ where

$$
a\left(\varepsilon, A_{0}^{\star}\right):=-\frac{\rho \pi}{2}\left(\lambda_{\varepsilon}+\varepsilon \gamma_{1}\left(0,0, A_{0}^{2}, \varepsilon\right)\right)<0, \quad b(\varepsilon):=\int_{0}^{+\infty} \gamma_{1}\left(u_{0}^{h}(\underline{x}), 0,0, \varepsilon\right) d \underline{x}
$$

and that our choice of $\varphi_{0}$ ensures that $\varphi \mapsto \Gamma_{\varphi}(\infty)$ is a monotonic bijection from ]$-\varphi_{0}, \varphi_{0}$ [ onto $] b(\varepsilon)-2 \pi, b(\varepsilon)+2 \pi\left[\right.$. So there exist three consecutive values $\varphi_{1}^{\star}<$ $\varphi_{2}^{\star}<\varphi_{3}^{\star}$ of $\varphi$ in $]-\varphi_{0}, \varphi_{0}\left[\right.$ and $n^{\star} \in \mathbb{Z}$ such that

$$
\Gamma_{\varphi_{1}^{\star}}(\infty)=\frac{\pi}{2}+n^{\star} \pi, \quad \Gamma_{\varphi_{2}^{\star}}(\infty)=\frac{\pi}{2}+\left(n^{\star}-1\right) \pi, \quad \Gamma_{\varphi_{3}^{\star}}(\infty)=\frac{\pi}{2}+\left(n^{\star}-2\right) \pi .
$$

Then, denote $c_{\star}=2 c_{2}$ and $\varepsilon_{\star}:=\min \left(\delta_{2}, 1 /\left(2 C_{2}\right)^{2}\right)$ and observe that for every $\left.\left.\alpha \in\right] 0, \frac{1}{2}\right]$, $\left.\varepsilon \in] 0, \varepsilon_{\star}\right]$ and every $A_{0}^{\star} \in\left[c_{\star} \varepsilon, \varepsilon^{1-\alpha}\right]$, we have $c_{\star} \varepsilon \leqslant \varepsilon^{1-\alpha}$ and 


$$
\begin{aligned}
& J\left(\mathfrak{h}_{1, A_{0}^{\star}, \varphi_{1}^{\star}, \varepsilon}, \varphi_{1}^{\star}, A_{0}^{\star}, \varepsilon\right) J\left(\mathfrak{h}_{1, A_{0}^{\star}, \varphi_{2}^{\star}, \varepsilon}, \varphi_{2}^{\star}, A_{0}^{\star}, \varepsilon\right) \leqslant-\left(c_{\star} e^{\frac{-\ell \lambda_{0}}{\varepsilon}}\right)^{2}<0, \\
& J\left(\mathfrak{h}_{1, A_{0}^{\star}, \varphi_{2}^{\star}, \varepsilon}, \varphi_{2}^{\star}, A_{0}^{\star}, \varepsilon\right) J\left(\mathfrak{h}_{1, A_{0}^{\star}, \varphi_{3}^{\star}, \varepsilon}, \varphi_{3}^{\star}, A_{0}^{\star}, \varepsilon\right) \leqslant-\left(c_{\star} \mathrm{e}^{\frac{-\ell \lambda_{0}}{\varepsilon}}\right)^{2}<0 .
\end{aligned}
$$

Hence, the intermediate value theorem ensures that for every $\left.\left.\left.\alpha \in] 0, \frac{1}{2}\right], \varepsilon \in\right] 0, \varepsilon_{\star}\right]$ and every $A_{0}^{\star} \in\left[c_{\star} \varepsilon, \varepsilon^{1-\alpha}\right]$ there are two distinct values of $\varphi$ in $]-\varphi_{0}, \varphi_{0}[$ denoted by $\varphi_{j}\left(\varepsilon, A_{0}^{\star}\right), j=1,2$, such that

$$
\begin{aligned}
& \left.\varphi_{1}\left(\varepsilon, A_{0}^{\star}\right) \in\right] \varphi_{1}^{\star}, \varphi_{2}^{\star}\left[, \quad \varphi_{2}\left(\varepsilon, A_{0}^{\star}\right) \in\right] \varphi_{2}^{\star}, \varphi_{3}^{\star}[, \\
& J\left(\mathfrak{h}_{1, A_{0}^{\star}, \varphi_{j}\left(\varepsilon, A_{0}^{\star}\right) \varepsilon}, \varphi_{j}\left(\varepsilon, A_{0}^{\star}\right), A_{0}^{\star}, \varepsilon\right)=0, \\
& 0<\varepsilon\left(\varphi_{2}\left(\varepsilon, A_{0}^{\star}\right)-\varphi_{1}\left(\varepsilon, A_{0}^{\star}\right)\right) \frac{\rho \pi}{2}\left(\frac{\lambda_{\varepsilon}}{\varepsilon}+\gamma_{1}\left(0,0, A_{0}^{2}, \varepsilon\right)\right)<2 \pi .
\end{aligned}
$$

This ensures that (9) admits two distinct reversible homoclinic connections $\mathrm{H}_{A_{0}, \varphi_{j}, \varepsilon}$ satisfying

$$
\mathrm{H}_{A_{0}, \varphi_{j}, \varepsilon}(\underline{x}) \underset{\underline{x} \rightarrow \pm \infty}{\longrightarrow} \mathrm{p}_{A_{0}, \varepsilon}\left(\underline{x} \pm \varepsilon \varphi_{j} \frac{\rho \pi}{2}\right),
$$

where $\mathrm{p}_{A_{0}, \varepsilon}$ is $T$-periodic with $T:=2 \pi /\left(\frac{\lambda_{\varepsilon}}{\varepsilon}+\gamma_{1}\left(0,0, A_{0}^{2}, \varepsilon\right)\right)$. The other phase shifts $\varphi$ for which $J$ vanishes lead to the same homoclinic connections.

\section{REFERENCES}

[1] C. Amick, On the theory of internal waves of permanent form in fluids of great depth, Trans. Amer. Math. Soc. 346 (1994) 399-419.

[2] C. Amick, J. Toland, Uniqueness and related analytic properties for the Benjamin-Ono equation - a nonlinear Neumann problem in the plane, Acta Math. 167 (1991) 107-126.

[3] T.B. Benjamin, Internal waves of permanent form in fluids of great depth, J. Fluid Mech. 29 (1967) 559-592.

[4] R.E. Davis, A. Acrivos, Solitary internal waves in deep water, J. Fluid Mech. 29 (1967) 593-607.

[5] F. Dias, G. Iooss, Water-Waves as a Spatial Dynamical System, Handbook of Mathematical Fluid Dynamics, to appear.

[6] G. Iooss, Gravity and capillary-gravity periodic travelling waves for two superposed fluid layers, one being of infinite depth, J. Math. Fluid Mech. 1 (1999) 24-61.

[7] G. Iooss, E. Lombardi, S.M. Sun, Gravity travelling waves for two superposed fluid layers, one being of infinite depth: a new type of bifurcation, Phil. Trans. R. Soc. London A 360 (2002) 2245-2336.

[8] T. Levi-Civita, Détermination rigoureuse des ondes permanentes d'ampleur finie, Math. Annalen 93 (1925) 264-314.

[9] E. Lombardi, Orbits homoclinic to exponentially small periodic orbits for a class of reversible systems. Application to water waves, Arch. Rat. Mech. Anal. 137 (1997) 227304.

[10] E. Lombardi, Oscillatory Integrals and Phenomena Beyond all Algebraic Orders, with Applications to Homoclinic Orbits in Reversible Systems, in: Lecture Notes in Math., Vol. 1741, Springer, 2000.

[11] H. Ono, Algebraic solitary waves in stratified fluids, J. Phys. Soc. Japan 39 (1975) 1082 1091. 
[12] E. Părău, F. Dias, Interfacial periodic waves of permanent form with free-surface boundary conditions, J. Fluid Mech. 437 (2001) 325-336.

[13] S.M. Sun, M.C. Shen, Exponentially small estimate for the amplitude of capillary ripples of a generalized solitary wave, J. Math. Anal. Appl. 172 (1993) 533-566.

[14] S.M. Sun, Existence of solitary internal waves in a two-layer fluid of infinite depth, Nonlinear Analysis 30 (8) (1997) 5481-5490.

[15] S.M. Sun, Nonexistence of truly solitary waves in water with small surface tension, Proc. Roy. London A 455 (1999) 2191-2228. 